\title{
Research on Shimmy Characteristics and Control Strategy of Double-Axle Steering Mechanism with Multiple Limit Cycles Considering Vehicle-Road Coupling
}

\author{
Yuhang Zhang \\ HFUT: Hefei University of Technology \\ Daogao Wei ( $\sim$ weidaogao@hfut.edu.cn ) \\ Heifei university of technology https://orcid.org/0000-0003-0875-7481 \\ Tong Jiang \\ HFUT: Hefei University of Technology \\ Bingzhan Zhang \\ HFUT: Hefei University of Technology \\ Wei Wang \\ HFUT: Hefei University of Technology
}

\section{Research Article}

Keywords: Vehicle-Road Coupling, Multiple Limit Cycles, Dual-axle Steering Mechanism, Shimmy, Control Strategy

Posted Date: August 17th, 2021

DOI: https://doi.org/10.21203/rs.3.rs-801053/v1

License: (c) (i) This work is licensed under a Creative Commons Attribution 4.0 International License.

Read Full License 


\title{
Research on Shimmy Characteristics and Control Strategy
}

\section{of Double-Axle Steering Mechanism with Multiple Limit}

\section{Cycles Considering Vehicle-Road Coupling}

\author{
Yuhang Zhang ${ }^{\mathrm{a}}$, Daogao Wei ${ }^{\mathrm{a}, *}$, Tong Jiang ${ }^{\mathrm{a}}$, Bingzhan Zhang ${ }^{\mathrm{a}}$, Wei Wang ${ }^{\mathrm{b}}$

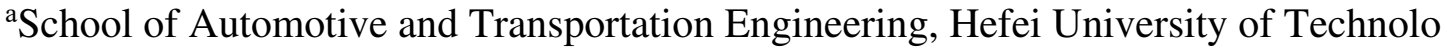 \\ gy, Hefei 23009, Anhui, China \\ ${ }^{\mathrm{b} S}$ School of Mechanical Engineering, Hefei University of Technology, Hefei 23009, A \\ nhui, China
}

\begin{abstract}
The vehicle and the road are a coupled system, the road surface characteristics have a great influence on the vehicle's handling stability and driving safety. So in this study, A 9-DOF mathematical model of dual-axle steering mechanism considering the vehicle-road coupling is established. Taking one multi-axle vehicle with dual-axle steering mechanism for example, the numerical calculation method is used to reproduce the multiple limit-cycles shimmy, and the effects of the road adhesion coefficient on the multiple limit-cycles shimmy characteristics is analyzed. The results show that the maximum swing angles of first and second axle and the speed ranges of multiple limit-cycles shimmy occurring decrease with the road adhesion coefficient decreasing, and as the road adhesion coefficient decreases, the speed range of multiple limit-cycles occurring tend to shrink to the middle speed range $(40-50 \mathrm{~km} / \mathrm{h})$ which is multi-axle vehicles often travelling. In addition, we also found that the multiple limit-cycles shimmy characteristic of the dual-axle steering mechanism is more sensitive to the change of the road adhesion coefficient of second axle than the change of the road adhesion coefficient of first axle. This finding can provide theoretical guidance for suppressing multiple limit-cycles shimmy of multi-axle vehicle with dual-axle steering mechanism. Finally, we propose a control strategy for the multiple limit cycle shimmy phenomenon, which reduces the amplitude of the shimmy and the interval of multiple limit cycles.
\end{abstract}

Keywords: Vehicle-Road Coupling; Multiple Limit Cycles; Dual-axle Steering Mechanism; Shimmy; Control Strategy

\section{Introduction}

Given large capacity, high horsepower, good performance, strong adaptive and other advantages, commercial multi-axle vehicle with dual-axle steering mechanism are widely utilized nowadays. The dual-axle steering system is an advanced steering system characterized by low cost, convenient operation, steady steering under heavy load and minimal damage to the road surface during driving. However, the vehicle shimmy sometimes occurs in dual-axle steering system during driving; this phenomenon causes abnormal wear of the tires, steering deviation and steering wheel wobbling [1-3]. Under a certain condition, shimmy with multiple limit-cycles can

* Corresponding author

Email address: weidaogao@hfut.edu.cn(Daogao Wei) 
occur in steering system. In this case, shimmy strength depends on the excitation value. A large shimmy excitation induces high-amplitude shimmy, and a small excitation induces low-amplitude shimmy. Shimmy with multiple limit-cycles causes the vehicle's traveling direction to become highly uncertain and increases the difficulty of controlling multi-axle vehicle. These conditions seriously affect the safety and stability of vehicle.

The shimmy of vehicle with a single-axle steering system has been studied for nearly 90 years, and can be divided into forced shimmy and self-excited shimmy. The self-excited shimmy is a Hopf bifurcation phenomenon. Extensive research has been conducted on this field and can be used to solve the shimmy problems in engineering [4-7]. Given the recent increase in market demand for multi-axle vehicle, current researches on dual-axle steering system have demonstrated its significance. Considering its sophisticated mechanism, the shimmy of a dual-axle steering system differs from that of a single-axle steering system. Therefore, many scholars have studied the problem extensively. Hou et al. [8] established kinematics and mathematical optimization models for the multi-axle steering system of a $10 \times 8$ multi-axle vehicle and designed a new weight function considering the probability of the steering angle. Wu and Hai [9] found the dual-axle could improve the yaw stability of a vehicle. Williams [10] extended the dual-axle model of vehicles to a multi-axle model, and analysed its steering and handing stability by establishing the differential equations of motion. Demi'c [11] utilized a linear dual-front axle yaw dynamics model to examine the influence of structural parameters on the front wheel shimmy of a heavy vehicle.Xiang Chen et al. [12] established a 3DOF mathematical model of the multi-axle steering mechanism of a multi-axle vehicle, and analyzed the causes of abnormal tire wear; Jinquan Ding and Konghui Guo [13] established a generalised system of equations to describe the fundamental handling dynamics of multi-axle vehicle including both the effects of vehicle body roll and multi-axle handling on vehicle dynamics. T Mi [14] established a 5-DOF EV shimmy model considering tire elasticity, and deduced the tyre-road constraint equation under the assumption of no slip at the leading edge contact point. The shimmy phenomena under different structural parameters and initial conditions are simulated numerically. The simulation results show that Hopf bifurcation will occur under the condition of a certain vehicle forward speed. X.G Li [15] built a 9-DOF model considering the coupled motion of the vehicle body on the basis of the 5-DOF system, and compared the two models, and found that the 9-DOF shimmy was more likely to occur in the low-speed zone. D.G Wei [16] established a mathematical model for dual-axle steering mechanism of a multi-axle vehicle considering dry friction to study the mechanism of self-excited shimmy of multi-axle vehicle with dual-axle steering mechanism. Based on the mathematical model established by D.G Wei, W Zhang [17] analyzed the influence of wheel positioning parameters on the shimmy characteristics of multi-limit cycle, and found that the influence of parameters on the shimmy characteristics of different vehicle Bridges was different. Z.B Wang [18] designed an adaptive robust control algorithm for the electro-hydraulic power steering system of a multi-axle vehicle, including steering mechanism, valvy-controlled dual hydraulic 
actuator and heavy-duty tire. The uncertain steering resistance moment of the tire was estimated online and the model uncertainty was compensated. The accurate tracking motion of EHPSS was realized by the establishment of a MATLAB simulation system. Q.M Zhang [19] simplifies the complex EHPSS mathematical model by using BP neural network, and conducts experimental verification of the model under no-load and load conditions, and finds that the system leakage has a great influence on the maintenance of working pressure.

In summary,a lot of studies focused on multi-axle vehicle with dual-axle steering mechanism, but few study focused on the mechanism of self-excited shimmy of steering system and multiple limit-cycles shimmy in self-excited.And the effects of vehicle-road coupling on shimmy system were not considered. The vehicle and the road are a coupled system, the road surface characteristics have a great influence on the vehicle's handling stability and driving safety. And the most intuitive performance of road surface characteristics is the road adhesion coefficient. Therefore, in order to more intuitively study the effects of vehicle-road coupling on multiple limit-cycles shimmy characteristics of dual-axle steering system, a 9-DOF dynamic model considering the road adhesion coefficient was built from the perspective of mechanism by using the Lagrangian equation. And taking one multi-axle vehicle with dual-axle steering mechanism we studied as an example to explore the effects of the vehicle-road coupling on multiple limit-cycles shimmy characteristics by numerical analysis. Finally, a control strategy for the shimmy phenomenon with multiple limit cycles is proposed, which reduces the amplitude of the shimmy and the interval of multiple limit cycles.

The innovative points in this study are as follows:

1. The vehicle and the road surface are a coupled system, the road surface characteristics have a great influence on the vehicle's handling stability and driving safety. And the most intuitive performance of road surface characteristics is the road adhesion coefficient. So a 9-DOF mathematical model of dual-axle steering mechanism considering the road adhesion coefficient was established;

2. Analyzed the influence of the road adhesion coefficient on the vibration characteristics of multiple limit cycles, and obtained the different influence rules of the road adhesion coefficient of the first and second bridges on the vibration characteristics of multiple limit cycles. The numerical simulation results reflect the physical phenomenon of the shimmy of the multi-axle car more truly.

3. A control strategy for the multi-limit cycle shimmy phenomenon of the steering wheel is proposed, and the system parameters that have a greater impact on the shimmy phenomenon and the optimal interval of each parameter are found. By matching appropriate parameters to the system, the pendulum is reduced. The vibration amplitude and multiple limit cycle intervals can achieve the purpose of parameter optimization.

4. The research on multiple limit-cycles is still a difficult in mathematics up to now. This paper finds the phenomenon of multiple limit-cycles by studying the shimmy of dual-axle steering mechanism. On the one hand, it can provide a set of differential equations which come from the engineering practice for studying the 
phenomenon of multiple limit-cycles in mathematics; On the other hand, the multiple limit-cycles shimmy mechanism of dual-axle steering mechanism is found preliminarily, which can provide a theoretical research method for the multiple limit-cycles vibration of similar mechanism in engineering.

\section{Model}

\subsection{Mechanical model and mathematical model}

A schematic of dual-axle steering mechanism is shown in Fig.1. The steering force generated by the turning of the steering wheel is transmitted to the steering gear through the steering operating mechanism (steering shaft, drive shaft, steering universal joint and so on). After reducing the speed and increasing the torque through the steering gear, the torque is transmitted to the first rocker. The steering knuckle arm of the first axle is driven to move by the movement of the anterior longitudinal rod. The left steering knuckle and the left wheel mounted on it rotate around the kingpin in the same direction. The left trapezoid arm causes the right wheel to rotate around the kingpin through the tie rod and the right trapezoid arm. Hence, the steering of the right wheel is in coordination with that of the left wheel. At the same time, the steering knuckle arms of the second axle move through the first transition rod, intermediate rocker, second transition rod, second rocker and posterior longitudinal rod. Hence, the steering of the wheels of the second axle is in coordination with that of the first axle. A schematic of the intermediate steering transmission mechanism is shown in Fig.2.

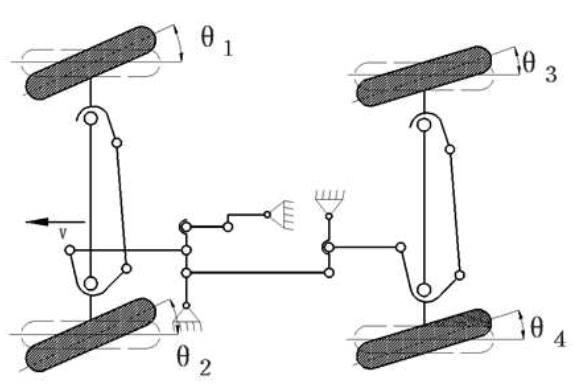

Fig.1. The schematic of dual-axle steering mechanism

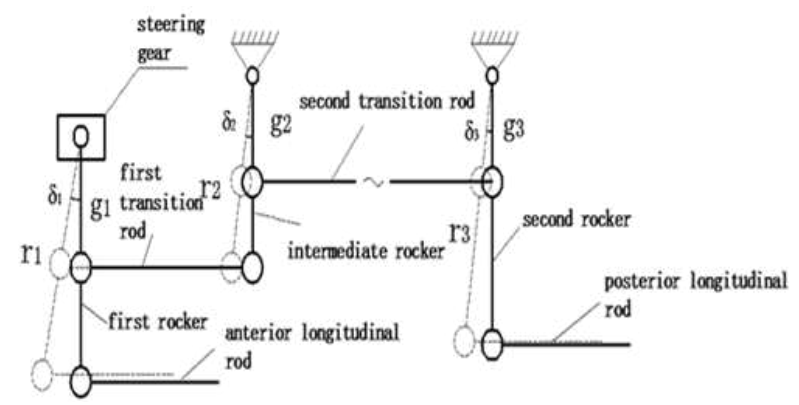

Fig. 2. The schematic of intermediate steering transmission mechanism 


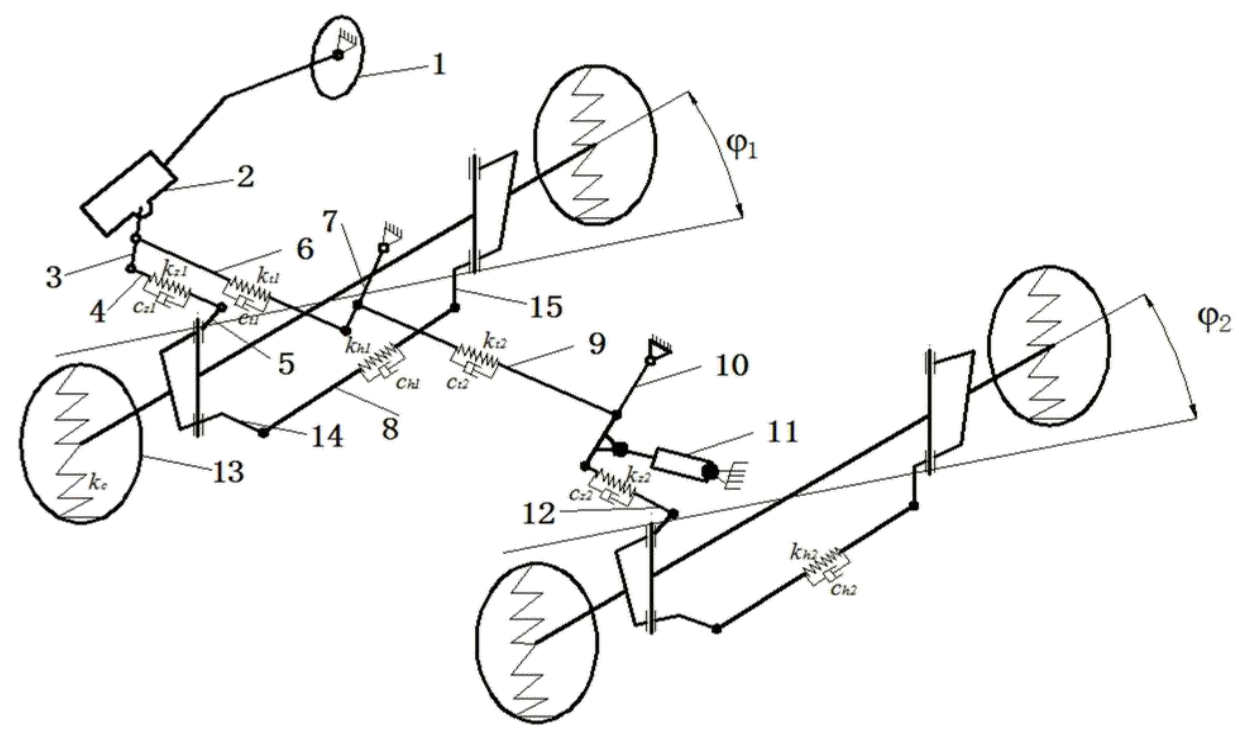

1.steering wheel 2.steering gear 3.first rocker 4.anterior longitudinal rod 5.steering knuckle arm 6.first transition rod 7.intermediate rocker 8.tie rod 9.second transition rod 10.second rocker 11.steering power cylinder 12.posterior longitudinal rod 13.tire 14.left trapezoid arm 15.right trapezoid arm

Fig.3. The dual-axle steering mechanism shimmy system

The mechanics model of dual-axle steering mechanism shimmy system is shown in Fig.3. In the mechanics model, the elastic of the rod is considered; it is equivalent to a spring-damper unit. In addition, the split road adhesion coefficients and the dry friction between the suspension and steering system are also considered when modeling. In line with the law of the right-hand coordinate system, the center of the mass of the vehicle is deemed as the coordinate origin; the vehicle forward direction is for $\mathrm{X}$-axis, the vehicle left direction is for $\mathrm{Y}$-axis, and perpendicular to the ground up direction is for Z-axis. According to Fig.3, the following assumptions are made when establishing the mechanical model of the dual-axle steering mechanism:

(1) The steering wheel is immobile;

(2) The impact of the force of air is ignored;

(3) The various parts associated with the vibration and their couplings are simplified according to the moments of inertia, springs, and dampers;

(4) The angle between the steering trapezoid plane and XY plane and the angle between the steering linkage and the $\mathrm{XZ}$ plane are ignored;

(5) The direction and speed of the vehicle is constant;

(6) Longitudinal and lateral slips do not occur in the vehicle.

The shimmy system has nine degrees of freedom: $\theta_{1}$ is the swing angle at which the right wheel of the first axle rotates around the kingpin; $\theta_{2}$ is the swing angle at which the left wheel of the first axle rotates around the kingpin; $\varphi_{1}$ is the lateral swing angle of the first axle; $\theta_{3}$ is the swing angle at which the right wheel of the second axle rotates around the kingpin; $\theta_{4}$ is the swing angle at which the left wheel of the second axle rotates around the kingpin; $\varphi_{2}$ is the lateral swing angle of the first axle; $\delta_{1}$ is the swing angle of the first rocker; $\delta_{2}$ is the swing angle of the intermediate rocker $; \delta_{3}$ is the swing angle of the second rocker. 
In this study, the mathematical model of the shimmy system is established by the Lagrange equation. which can be expressed as:

$$
\frac{d}{d t}\left(\frac{\partial T}{\partial \dot{q}_{k}}\right)-\frac{\partial T}{\partial q_{k}}+\frac{\partial U}{\partial q_{k}}+\frac{\partial D}{\partial \dot{q}_{k}}=Q_{k}
$$

where $q_{k}$ represents nine degrees of freedom of the system, $T$ represents the system's kinetic energy, $U$ represents the system's potential energy, $D$ represents the system's dissipated energy, and $Q_{k}$ represents the nine generalized forces to which the system is subjected.

According to Fig.3, kinetic energy, potential energy, dissipated energy, and the nine generalized forces of the dual-axle steering shimmy system are obtained as follows.

The kinetic energy of the shimmy system is

$$
\begin{aligned}
& T=\frac{1}{2} I_{1}\left(\dot{\theta}_{1}^{2}+\dot{\theta}_{2}^{2}\right)+\frac{1}{2} J_{1} \dot{\varphi}_{1}^{2}+i_{k 1} \frac{v}{R} \sin \varphi_{1}\left(\dot{\theta}_{1}+\dot{\theta}_{2}\right)+\frac{1}{2} I_{2}\left(\dot{\theta}_{3}^{2}+\dot{\theta}_{4}^{2}\right)+\frac{1}{2} J_{2} \dot{\varphi}_{2}^{2} \\
& +\frac{1}{2} I_{c 1} \dot{\delta}_{1}^{2}+\frac{1}{2} I_{c 2} \dot{\delta}_{2}^{2}+\frac{1}{2} I_{c 3} \dot{\delta}_{3}^{2}+i_{k 2} \frac{v}{R} \sin \varphi_{2}\left(\dot{\theta}_{3}+\dot{\theta}_{4}\right)
\end{aligned}
$$

where $i_{k i}(\mathrm{i}=1,2)$ is the moment of inertia of the wheel around its own axis of rotation in ith axle, $I_{i}(i=1,2)$ is the moment of inertia of the wheel around the kingpin in $i$ th axle, $I_{c i}(i=1,3)$ is the moment of inertia of $i t h$ rocker, $I_{c 2}$ is the moment of inertia of intermediate rocker, and $J_{i}(i=1,2)$ is the moment of inertia of ith axle around its side off-axis.

The potential energy of the shimmy system is

$$
\begin{aligned}
& U=\frac{1}{2} k_{h 1}\left(\theta_{1}-\theta_{2}\right)^{2}+\frac{1}{2} k_{n} \varphi_{1}^{2}+\frac{1}{2} k_{h 2}\left(\theta_{3}-\theta_{4}\right)^{2}+\frac{1}{2} k_{n} \varphi_{2}^{2} \\
& +\frac{1}{2} k_{t} \delta_{1}^{2}+\frac{1}{2} k_{t 1}\left(g_{1} \delta_{1}-r_{2} \delta_{2}\right)^{2}+\frac{1}{2} k_{t 2}\left(g_{2} \delta_{2}-g_{3} \delta_{3}\right)^{2} \\
& +\frac{1}{2} k_{z 1}\left(r_{1} \delta_{1}+a_{1} \theta_{2}\right)^{2}+\frac{1}{2} k_{z 2}\left(r_{3} \delta_{3}+a_{2} \theta_{4}\right)^{2}
\end{aligned}
$$

where $k_{h i}(i=1,2)$ is the stiffness of the tie rod converted into the stiffness around the kingpin, $k_{n}$ is the equivalent angle stiffness of the suspension converted into the side swing center, $k_{t}$ is the inverse stiffness of the first rocker to the steering wheel, $k_{t i}(i=1,2)$ is the stiffness of $i t h$ transition $\operatorname{rod}, k_{z i}(i=1,2)$ is the stiffness of the anterior and posterior longitudinal rods, $r_{l}$ is the effective length of the first rocker, $r_{2}$ is the effective length of intermediate rocker, $r_{3}$ is the effective length of the second rocker $g_{1}, g_{2}$ and $g_{3}$ are the distances between the pivot of the rocker and the transition lever and the fixed end of the rocker arm, and $a_{i}(i=1,2)$ is the distance between the hinge point of $i$ th axle knuckle arm and vertical rod and kingpin.

$$
\begin{aligned}
D= & \left.\left.\frac{1}{2} c_{l 1} \dot{\theta}_{1}^{2}+\dot{\theta}_{2}^{2}\right)+\frac{1}{2} c_{h 1} \dot{\theta}_{1}-\dot{\theta}_{2}\right)^{2}+\frac{1}{2} c_{n} \dot{\varphi}_{1}^{2}+\frac{1}{2} c_{h 2}\left(\dot{\theta}_{3}-\dot{\theta}_{4}\right)^{2}+\frac{1}{2} c_{l 2}\left(\dot{\theta}_{3}^{2}+\dot{\theta}_{4}^{2}\right)+\frac{1}{2} c_{n} \dot{\varphi}_{2}^{2} \\
& +\frac{1}{2} c_{t} \dot{\delta}_{1}^{2}+\frac{1}{2} c_{t 1}\left(g_{1} \dot{\delta}_{1}-r_{2} \dot{\delta}_{2}\right)^{2}+\frac{1}{2} c_{t 2}\left(g_{2} \dot{\delta}_{2}-g_{3} \dot{\delta}_{3}\right)^{2}+\frac{1}{2} c_{z 1}\left(r_{1} \dot{\delta}_{1}+a_{1} \dot{\theta}_{2}\right)^{2} \\
& +\frac{1}{2} c_{z 2}\left(r_{3} \dot{\delta}_{3}+a_{2} \dot{\theta}_{4}\right)^{2}+M_{f 1} \dot{\theta}_{1}+M_{f 2} \dot{\theta}_{2}+M_{f 3} \dot{\theta}_{3}+M_{f 4} \dot{\theta}_{4}
\end{aligned}
$$


Where $c l i(i=1,2)$ is the equivalent damping of $i t h$ wheel around the kingpin, $c h i$ $(i=1,2)$ is the damping of the tie rod converted into the damping around the kingpin, $c_{n}$ is the equivalent damping of the suspension converted into the side swing center, $c_{t}$ is the inverse damping of the first rocker to the steering wheel, $c_{t i}(\mathrm{i}=1,2)$ is the damping of $i$ th transition rod, $c_{z i}(i=1,2)$ is the damping of the anterior and posterior longitudinal rods, $M_{f l}$ is the equivalent friction torque of the first axle at the right wheel kingpin, $M_{f 2}$ is the equivalent friction torque of the first axle at the left wheel kingpin, $M_{f 3}$ is the equivalent friction torque of the second axle at the right wheel kingpin, $M_{f 4}$ is the equivalent friction torque of the second axle at the left wheel kingpin.

The nine generalized forces of the shimmy system are as follows:

$$
\begin{aligned}
& Q_{1}=F_{y 1}(R \gamma+e)-\left(\gamma \rho h_{1} R+\frac{k_{\mathrm{c}}}{2} B_{1} l_{1}(-f+\gamma)\right) \varphi_{1} \\
& Q_{2}=F_{y 2}(R \gamma+e)-\left(\gamma \rho h_{1} R+\frac{k_{c}}{2} B_{1} l_{1}(-f+\gamma)\right) \varphi_{1} \\
& Q_{3}=-\left(2 \rho R h_{1}^{2}+\frac{1}{2} k_{c} B_{1}^{2}\right)+\left(F_{y 1}+F_{y 2}\right) h_{1} \\
& Q_{4}=F_{y 3}(R \gamma+e)-\left(\gamma \rho h_{2} R+\frac{k_{\mathrm{c}}}{2} B_{2} l_{2}(-f+\gamma)\right) \varphi_{2} \\
& Q_{5}=F_{y 4}(R \gamma+e)-\left(\gamma \rho h_{2} R+\frac{k_{\mathrm{c}}}{2} B_{2} l_{2}(-f+\gamma)\right) \varphi_{2} \\
& Q_{6}=-\left(2 \rho R h_{2}{ }^{2}+\frac{1}{2} k_{c} B_{2}{ }^{2}\right)+\left(F_{y 3}+F_{y 4}\right) h_{2} \\
& Q_{7}=0, Q_{8}=0, Q_{9}=0
\end{aligned}
$$

Where $v$ is the vehicle speed, $R$ is the rolling radius of the tire, $F_{y l}$ is the first axle right wheel subjected to lateral force, $F_{y 2}$ is the first axle left wheel subjected to lateral force, $F_{y 3}$ is the second axle right wheel subjected to lateral force, $F_{y 4}$ is the second axle left wheel subjected to lateral force, $e$ is the pneumatic trail, $\rho$ is the tire lateral stiffness, $h_{i}(i=1,2)$ is the height of $i t h$ suspension roll center, $k_{c}$ is the tire vertical stiffness, $B_{i}(i=1,2)$ is the tread in $i t h$ axle, $l_{i}(i=1,2)$ is the distance between the point of the kingpin extension line with the ground intersection and plane of symmetry of the wheel, $f$ is the friction coefficient between the tire and the ground.

According to (1) to (5), the kinetic equations for the system are derived from Lagrangian equations. Because the value of $\varphi_{1}$ and $\varphi_{2}$ is very small, $\cos \varphi_{1} \approx 1, \cos \varphi_{2} \approx 1$. The simplified differential equations are as follows: 


$$
\begin{aligned}
& I_{1} \ddot{\theta}_{1}+c_{l 1} \dot{\theta}_{1}+k_{h 1}\left(\theta_{1}-\theta_{2}\right)+c_{h 1}\left(\dot{\theta}_{1}-\dot{\theta}_{2}\right)+\frac{i_{k 1} v}{R} \dot{\varphi}_{1}+\left(\rho R h_{1} \gamma+\frac{1}{2} k_{c} l_{1} B_{1}(\gamma-f)\right) \varphi_{1} \\
& -F_{y 1}(R \gamma+e)+M_{f 1}=0 \\
& I_{1} \ddot{\theta}_{2}+c_{l 1} \dot{\theta}_{2}+k_{h 1}\left(\theta_{2}-\theta_{1}\right)+c_{h 1}\left(\dot{\theta}_{2}-\dot{\theta}_{1}\right)+k_{z 1} a_{1}\left(r_{1} \delta_{1}+a_{1} \theta_{2}\right) \\
& +c_{z 1} a_{1}\left(r_{1} \dot{\delta}_{1}+a_{1} \dot{\theta}_{2}\right)+\frac{i_{k 1} v}{R} \dot{\varphi}_{1}+\left(\rho R h_{1} \gamma+\frac{1}{2} k_{c} l_{1} B_{1}(\gamma-f)\right) \varphi_{1} \\
& -F_{y 2}(R \gamma+e)+M_{f 2}=0 \\
& J_{1} \ddot{\varphi}_{1}+c_{n 1} \dot{\varphi}_{1}+\left(\frac{1}{2} k_{c} B_{1}^{2}+k_{n}+2 \rho h_{1}^{2}\right) \varphi_{1}-\frac{i_{k 1} v}{R}\left(\dot{\theta}_{1}+\dot{\theta}_{2}\right)+\left(F_{y 1}+F_{y 2}\right) h_{1}=0 \\
& I_{2} \ddot{\theta}_{3}+c_{l 2} \dot{\theta}_{3}+k_{h 2}\left(\theta_{3}-\theta_{4}\right)+c_{h 2}\left(\dot{\theta}_{3}-\dot{\theta}_{4}\right)+\frac{i_{k 2} v}{R} \dot{\varphi}_{2} \\
& +\left(\rho R h_{2} \gamma+\frac{1}{2} k_{c} l_{1} B_{2}(\gamma-f)\right) \varphi_{2}-F_{y 3}(R \gamma+e)+M_{f 3}=0 \\
& I_{2} \ddot{\theta}_{4}+c_{l 2} \dot{\theta}_{4}+k_{h 2}\left(\theta_{4}-\theta_{3}\right)+c_{h 2}\left(\dot{\theta}_{4}-\dot{\theta}_{3}\right)+k_{z 2} a_{2}\left(r_{3} \delta_{3}+a_{2} \theta_{4}\right)+ \\
& c_{z 2} a_{2}\left(r_{3} \dot{\delta}_{3}+a_{2} \dot{\theta}_{4}\right)+\frac{i_{k 2} v}{R} \dot{\varphi}_{2}+\left(\rho R h_{2} \gamma+\frac{1}{2} k_{c} l_{2} B_{2}(\gamma-f)\right) \varphi_{2} \\
& -F_{y 4}(R \gamma+e)+M_{f 4}=0 \\
& J_{2} \ddot{\varphi}_{2}+c_{n 2} \dot{\varphi}_{2}+\left(\frac{1}{2} k_{c} B_{2}{ }^{2}+k_{n}+2 \rho h_{2}^{2}\right) \varphi_{2}-\frac{i_{k 2} v}{R}\left(\dot{\theta}_{3}+\dot{\theta}_{4}\right)+\left(F_{y 3}+F_{y 4}\right) h_{2}=0 \\
& I_{c 1} \ddot{\delta}_{1}+c_{t} \dot{\delta}_{1}+k_{t} \delta_{1}+c_{t 1} g_{1}\left(g_{1} \dot{\delta}_{1}-r_{2} \dot{\delta}_{2}\right)+k_{t 1} g_{1}\left(g_{1} \delta_{1}-r_{2} \delta_{2}\right)+c_{z 1} r_{1}\left(r_{1} \dot{\delta}_{1}+a_{1} \dot{\theta}_{2}\right) \\
& +k_{z 1} r_{1}\left(r_{1} \delta_{1}+a_{1} \theta_{2}\right)=0 \\
& I_{c 2} \ddot{\delta}_{2}+c_{t 1} r_{2}\left(r_{2} \dot{\delta}_{2}-g_{1} \dot{\delta}_{1}\right)+k_{t 1} r_{2}\left(r_{2} \delta_{2}-g_{1} \delta_{1}\right)+c_{t 2} g_{2}\left(g_{2} \dot{\delta}_{2}-g_{3} \dot{\delta}_{3}\right) \\
& +k_{t 2} g_{2}\left(g_{2} \delta_{2}-g_{3} \delta_{3}\right)=0 \\
& I_{c 3} \ddot{\delta}_{3}+c_{t 2} g_{3}\left(g_{3} \dot{\delta}_{3}-g_{2} \dot{\delta}_{2}\right)+k_{t 2} g_{3}\left(g_{3} \delta_{3}-g_{2} \delta_{2}\right)+c_{z 2} r_{3}\left(r_{3} \dot{\delta}_{3}+a_{2} \dot{\theta}_{4}\right) \\
& +k_{z 2} r_{3}\left(r_{3} \delta_{3}+a_{2} \theta_{4}\right)=0
\end{aligned}
$$

\subsection{Dry friction model}

Coulomb, Stribeck, Bouc-Wen and hysteresis loop are commonly used fry-friction models. Among these models, the Stribeck model can best reflect the nonlinear relationship between the relative speeds of two objects in contact with a dry-friction coefficient; the model also has good continuity. Moreover, using the Stribeck model to analyse this situation qualitatively is convenient because of good 
continuity. Hence, in this study, the Stribeck model with a negative slope characteristic [20]was selected for the calculation analysis. The mathematical expression of this model is

$$
\mu\left(\omega_{r}\right)=\left(\mu_{k}+\left(\mu_{s}-\mu_{k}\right) e^{-\kappa\left|\omega_{r}\right|}\right) \operatorname{sign}\left(\omega_{r}\right)
$$

In order to reduce the time in simulation, the dry friction model curve in Equation.(15) is smoothed; and the sign function is changed to the tanh function. Hence, the mathematical expression for the approximate Stribeck model is

$$
\mu\left(\omega_{r}\right)=\mu_{k}\left(1.0+(1 / \xi-1.0) e^{-\kappa\left|\omega_{r}\right|} \tanh \left(\sigma_{r} \omega_{r}\right)\right.
$$

Where $\omega_{r}$ is the relative velocity between two contact objects, $\kappa$ is the exponential decay factor that presents the negative slope value of dry friction (when $\kappa$ increases, the attenuation change in $\mu\left(\omega_{r}\right)$ versus $\omega$ becomes highly severe), $\sigma_{r}$ is the sliding parameter, $\mu_{k}$ is the dynamic friction coefficient, $\mu_{s}$ is the static friction coefficient and $\zeta=\mu_{k} / \mu_{s}$ is the ratio of the dynamic and static friction coefficient. Among them, take $\kappa=4.6, \sigma_{r}=50, \mu_{k}=0.3$ and $\mu_{s}=0.5$. The relation curve between $\mu\left(\omega_{r}\right)$ and $\omega_{r}$ is shown in Fig. 4.

The dry friction torque of the system is

$$
M_{f i}=\mu\left(\omega_{r}\right) M \quad(\mathrm{i}=1,2,3,4)
$$

where $M$ is the amplitude of dry friction torque.

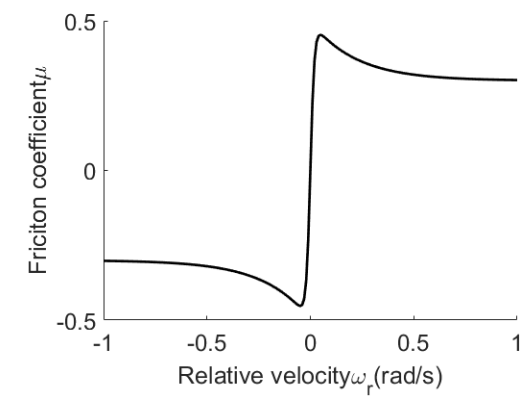

Fig.4. Curve of the dry-friction coefficient versus relative velocity

\subsection{Tire model}

The lateral force can be expressed as a simplified 'magic formula' [21-22]. The mathematical expression for the lateral force can be expressed as follows.

$$
F_{y i}=D \sin \left(\left(C \operatorname{atan}\left(B(1-E) \alpha_{i}+E \operatorname{atan}\left(B \cdot \alpha_{i}\right)\right)\right),\right.
$$

With

$$
\begin{aligned}
& D=a_{1} F_{Z}^{2}+a_{2} F_{Z} \\
& B=\frac{a_{3} \sin \left(a_{4}\left(\operatorname{atan}\left(a_{5} F_{Z}\right)\right)\right)}{C \cdot D} \\
& E=a_{6} F_{Z}^{2}+a_{7} F_{Z}+a 8 \\
& C=1.3
\end{aligned}
$$

Where $B$ is stiffness in the magic formula, $C$ is the shape in the magic formula, $D$ is the peak in the magic formula, $E$ is the curvature factor in the magic formula 
$a_{1}, a_{2}, a_{3}, a_{4}, a_{5}, a_{6}, a_{7}, a_{8}$ are the parameters obtained by experimental fitting, $F_{z}$ is the load on the tire, $\alpha_{i}(i=1,2,3,4)$ is the slip angle of the tire.Table.1 lists the fitting parameters for the calculation of the tire lateral force.

Table.1. Magic formula parameters

\begin{tabular}{ccccccccc}
\hline Parameter & $a_{1}$ & $a_{2}$ & $a_{3}$ & $a_{4}$ & $a_{5}$ & $a_{6}$ & $a_{7}$ & $a_{8}$ \\
\hline Value & -22.1 & 1011 & 1078 & 1.82 & 0.208 & 0 & -0.354 & 0.707 \\
\hline
\end{tabular}

The model has a high accuracy. Therefore, this model is suitable for calculation and analysis of the shimmy system. However, Equation.(18) does not explicitly represent the road adhesion coefficient. To study the effect that the road adhesion coefficients have on the shimmy characteristics, several parameters are modified to indicate the road conditions [23]. The improved magic formula can be described by

$$
F_{y i}=D^{*} \sin \left(\left(C^{*} \operatorname{atan}\left(B^{*}\left(1-E^{*}\right) \alpha_{i}+E^{*} \operatorname{atan}\left(B^{*} \cdot \alpha_{i}\right)\right)\right)\right.
$$

where

$$
B^{*}=\left(2-\mu_{i}\right) B, C^{*}=\frac{5-\mu_{i}}{4} C, D^{*}=\mu_{i} D, E^{*}=E
$$

Thus, equation (19) can be improved to give

$$
\begin{aligned}
& F_{y i}=\mu_{i} D \sin \left(\left(5 / 4-\mu_{i} / 4\right) C \operatorname{atan}\left(\left(2-\mu_{i}\right) B(1-E) o_{i}+E \operatorname{atan}\left(B \alpha_{i}\right)\right)\right) \\
& (i=1,2,3,4)
\end{aligned}
$$

Where $\mu_{1}$ is the road adhesion coefficient of the right wheel of the first bridge, $\mu_{2}$ is the road adhesion coefficient of the left wheel of the first bridge, $\mu_{3}$ is the road adhesion coefficient of the right wheel of the second bridge, $\mu_{4}$ is the road adhesion coefficient of the left wheel of the second bridge.

Fig.5 shows the relationship between lateral force $F_{y i}$ and $\alpha_{i}$ when the road adhesion coefficient $\mu_{i}$ is constant $\left(\mu_{i}=0.95\right)$ and the vertical load $F_{z}=4214 \mathrm{~N}$.

Fig. 6 shows the three-dimensional relationship along the $F_{y i}, \alpha_{i}$ and $\mu_{i}$ axes.

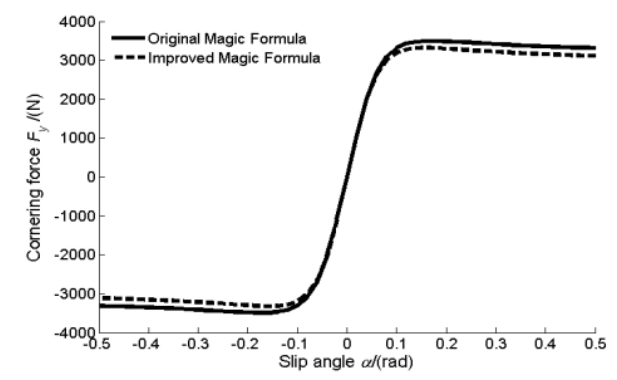

Fig.5. Curve of relationship between Fyi and $\alpha i$ 


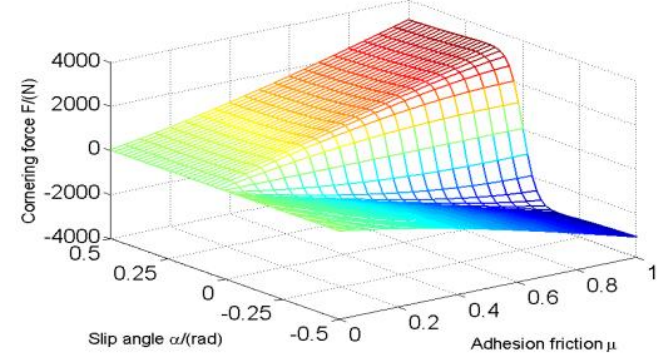

Fig.6. Slip angle and $\alpha_{i}$ road adhesion coefficient $\boldsymbol{\mu}_{\boldsymbol{i}}$ : curves of the lateral force $\boldsymbol{F}_{\boldsymbol{y} \boldsymbol{i}}$

To study the dynamic characteristics of the swing angle of the wheel, Qian et al. [24] used a non-holonomic constraint equation for a rolling tire to characterize the relationship between the slip angle of the tire and the swing angle of the wheel as

$$
\dot{\alpha}_{i}+\frac{v}{\sigma} \alpha_{i}+\frac{v}{\sigma} \theta_{i}-\frac{e_{i}}{\sigma} \dot{\theta}_{i}=0, \quad i=1,2,3,4
$$

where $\sigma$ is the relaxation length of the tire; $e_{i}(i=1,2,3,4)$ is the half-length of the contact area of the tire.

\section{Result and Discussion}

According to a multi-axle vehicle with dual-axle steering mechanism we studied, the structural parameters and partial stiffness in the mathematical model are determined. And some stiffness parameters and damping parameters are determined with reference to relevant data. Under the condition that other parameters are unchanged except the road adhesion coefficients, the effects of road adhesion coefficient on multiple limit-cycles shimmy characteristics have be discussed. The parameters of the sample mechanism in calculation are shown in Table 2.

Table.2. Parameters of the sample vehicle in calculation

\begin{tabular}{|c|c|c|c|}
\hline Parameter & Value & Parameter & Value \\
\hline$I_{1}, I_{2} / \mathrm{kg} \cdot \mathrm{m}^{2} \cdot \mathrm{rad}^{-}$ & 21.16 & $k_{z 1}, k_{z 2} / N \cdot \mathrm{rad}^{-}$ & $8 \mathrm{e} 5$ \\
\hline$J_{1,} J_{2} / \mathrm{kg} \cdot \mathrm{m}^{2} \cdot \mathrm{rad}$ & 210.8 & $c_{z 1}, c_{z 2} / N . m . s$. & 30 \\
\hline$I_{c 1} / \mathrm{kg} \cdot \mathrm{m}^{2} \cdot \mathrm{rad}^{-1}$ & 0.38 & $k_{t 1}, k_{t 2} / N . m^{-1}$ & $5.6 \mathrm{e} 5$ \\
\hline$I_{c 2} / \mathrm{kg} \cdot \mathrm{m}^{2} \cdot \mathrm{rad}^{-1}$ & 0.22 & $c_{t 1}, c_{t 2} / N . s$ & 15 \\
\hline$I_{c 3} / \mathrm{kg} \cdot \mathrm{m}^{2} \cdot \mathrm{rad}^{-1}$ & 0.28 & $k_{c} / N_{r a d}{ }^{-1}$ & $1.06 \mathrm{e} 6$ \\
\hline$i_{k 1}, i_{k 2} / \mathrm{kg} \cdot \mathrm{m}^{2} \cdot r a$ & 17.2 & $f$ & 0.02 \\
\hline$k_{h 1}, k_{h 2} / N \cdot r a d^{-1}$ & 37790 & $k_{t} / N . \mathrm{rad}^{-1}$ & 21000 \\
\hline ch1,Ch2/N.m.s.r & 35 & $c_{t} / N . m . s . r a d^{-}$ & 10 \\
\hline cll,cl2/N.m.s.ra & 105 & $\rho / N \cdot \operatorname{rad}^{-1}$ & 668000 \\
\hline$k_{n} / N \cdot r a d^{-1}$ & 31360 & $R / m$ & 0.52 \\
\hline
\end{tabular}




\begin{tabular}{|c|c|c|c|}
\hline$c_{n} /$ N.m.s.rad ${ }^{-1}$ & 1029 & $e / m$ & 0.05 \\
\hline$\gamma_{1}, \gamma_{2} /^{\circ}$ & 2.5 & $h_{1}, h_{2} / m$ & 0.45 \\
\hline$l_{1}, l_{2} / m$ & 0.07 & $B_{1,} B_{2} / m$ & 2.06 \\
\hline$r_{1} / m$ & 0.34 & $r_{2} / m$ & 0.42 \\
\hline$r_{3} / m$ & 0.47 & $g_{1} / m$ & 0.17 \\
\hline$g_{2} / m$ & 0.27 & $g_{3} / m$ & 0.22 \\
\hline$a_{1} / m$ & 0.22 & $a_{2} / m$ & 0.22 \\
\hline$\sigma / m$ & 1.216 & $e_{1}, e_{2}, e_{3,}, e_{4}$ & 0.02 \\
\hline
\end{tabular}

\subsection{Numerical calculation}

Taking $V=65 \mathrm{~km} / \mathrm{h}, \mu 1=\mu 2=\mu 3=\mu 4=0.8$, Based on the above differential equation (6) (21) of the shimmy system, the Runge-Kutta method was used to conduct numerical simulation, and the shimmy phase diagram of multiple limit cycles around the first and second Bridges was obtained, as shown in Fig. 7.

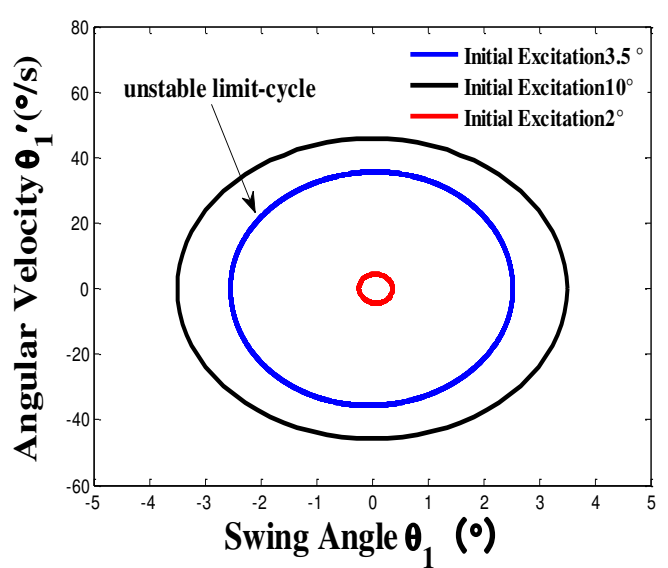

(a)right wheel of the first axle

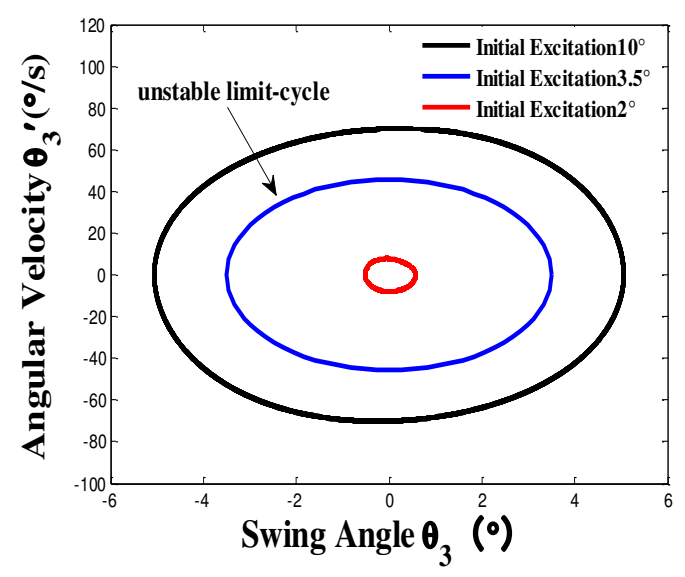

(c)right wheel of the second axle

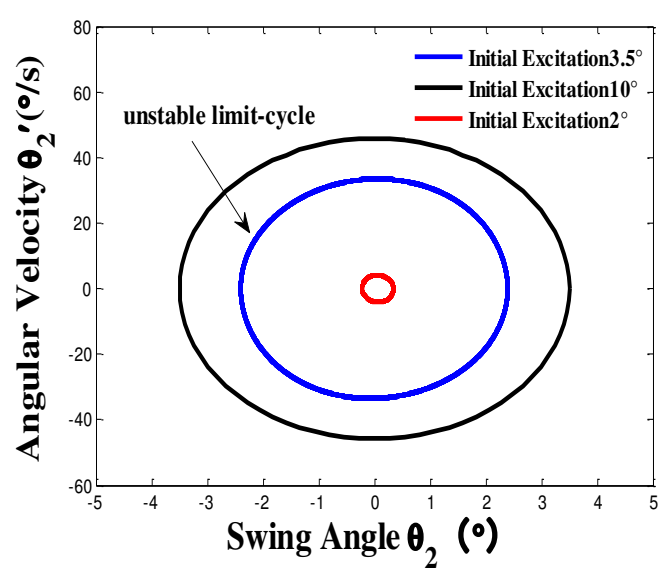

(b) left wheel of the first axle

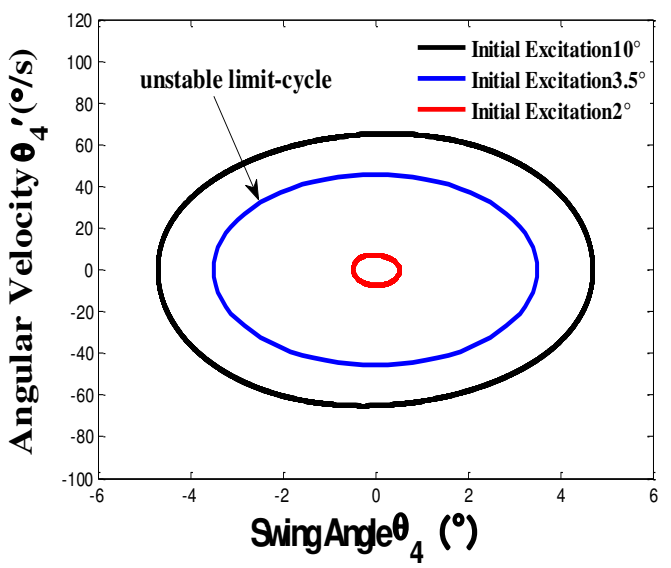

(d) left wheel of the second axle

Fig.7. Shimmy phase diagrams when $v=65 \mathrm{~km} / \mathrm{h}$

It can be seen from Fig. 7 that, when the initial excitation is $2^{\circ}$, the left and right wheels of the first and second axle show a marginal limit-cycle shimmy. When the initial excitation is $3.5^{\circ}$, the shimmy of the left and right wheels of the first and 
second axles is in the form of unstable limit -cycles. In this case, the system shimmy characteristics are unstable, and abrupt changes occur between the shimmy of small value and that of large value. When the initial excitation is increased to $10^{\circ}$, the system shimmy is characterized by a large and stable limit-cycle shimmy. Fig. 7 shows that the steering shimmy system of the dual-axle presents multi limit-cycle shimmy under the condition of the split road. The shimmy strength of the multi limit-cycle shimmy depends on the size of the initial excitation, and when the initial excitation exceeds a certain value, large amplitude shimmy can be induced. By comparing Fig. 7(a) with Fig. 7(c), under the same conditions, the pendulum angle amplitude of the second axle is obviously greater than that of the first axle, which is consistent with the actual usage of double-axle heavy truck. In reality, the wear of the two-axle tire is always more serious than that of the one-axle tire, which proves the reliability of the 9-DOF dual-axle steering system established in this paper.

\subsection{Effect of the road adhesion coefficients on multiple limit-cycles shimmy characteristics of dual-axle steering mechanism}

With the mathematical model established for the sample mechanism, the effects of road adhesion coefficients on multiple limit-cycles shimmy characteristics were calculated in Runge-Kutta method. Taking $\mu_{1}=\mu_{2}=\mu_{3}=\mu_{4}=0.8$, the global bifurcation diagrams of swing angles of the first and second axle versus speed were obtained, as shown in Fig.8.Taking $v=70 \mathrm{~km} / \mathrm{h}$, the multiple limit-cycles shimmy phase diagram of the first and second axle were obtained, as shown in Fig.9.

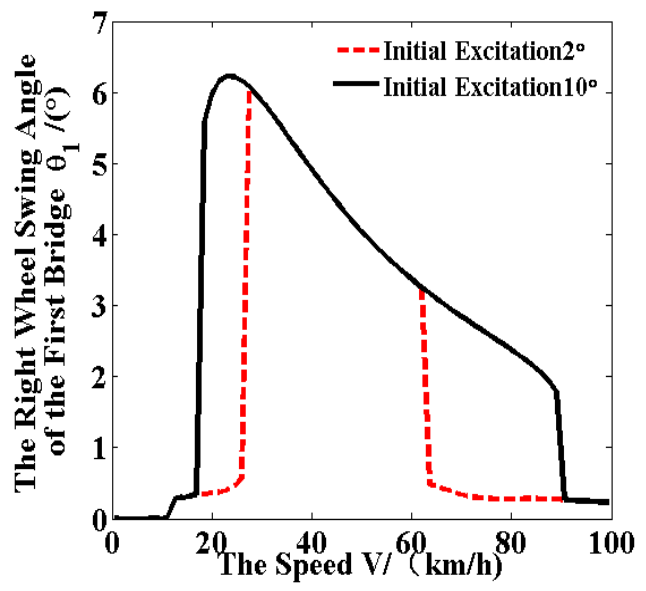

(a) right wheel of the first axle

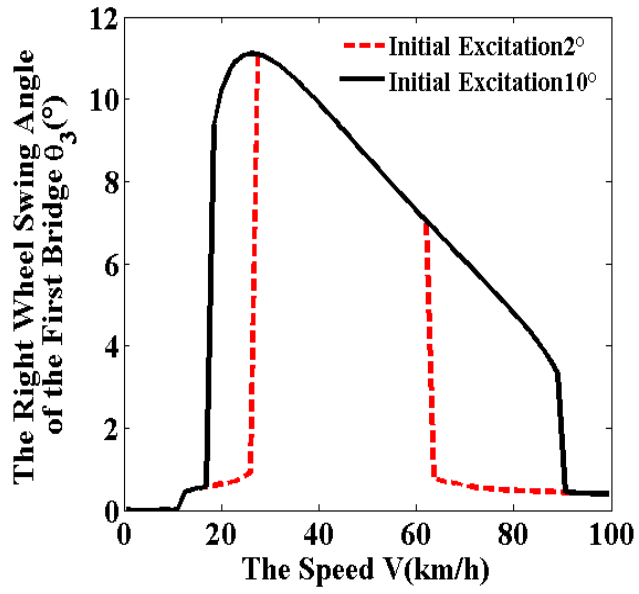

(b) right wheel of the second axle

Fig.8. Bifurcation diagrams of the shimmy system when $\mu_{1}=\mu_{2}=\mu_{3}=\mu_{4}=0.8$ 


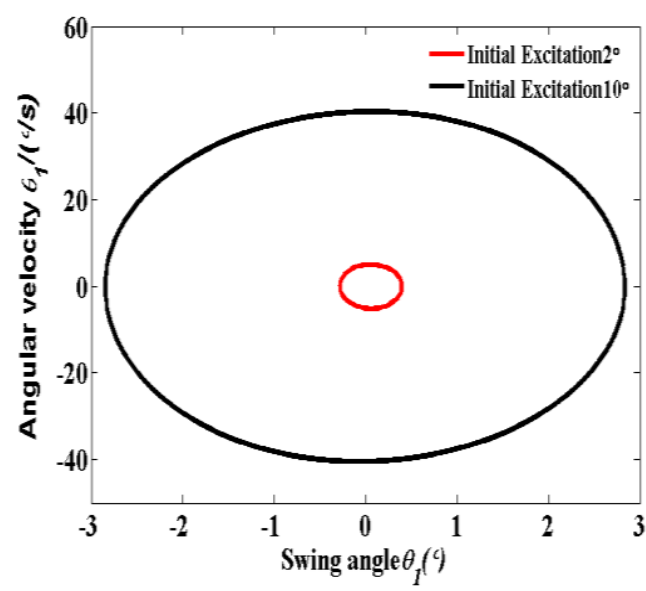

(a)right wheel of the first axle

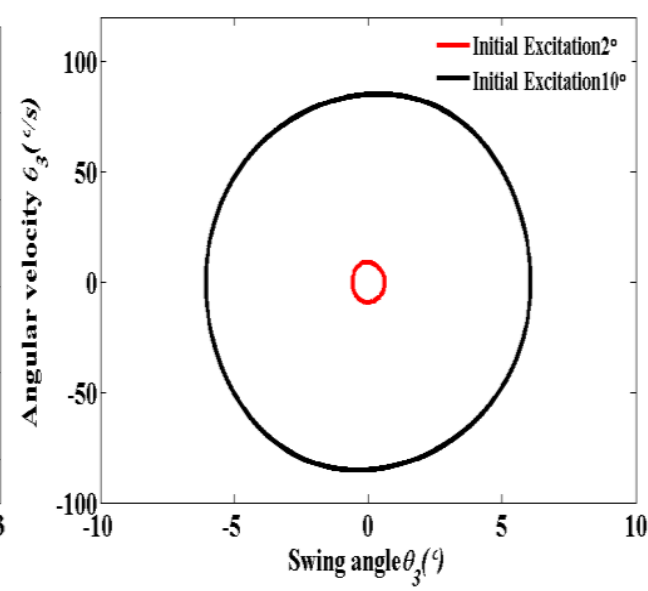

(b) right wheel of the second axle

Fig.9. Shimmy phase diagrams when $v=70 \mathrm{~km} / \mathrm{h}$

It can be seen from Fig. 8 that the dual-axle steering mechanism produces shimmy under a certain initial excitation, and the amplitude of shimmy induced changes with the change of vehicle speed. As shown in Fig. 7(a), within the velocity range [27.62] $\mathrm{km} / \mathrm{h}$, the amplitude of oscillation Angle of different excitation laps is

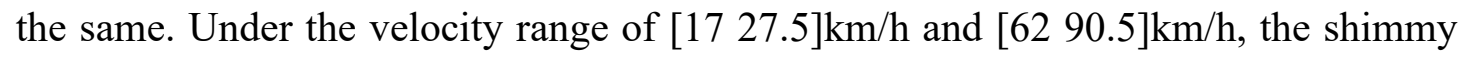
Angle amplitude induced by different excitation is also different. Under the small initial excitation $\left(2^{\circ}\right)$, the shimmy with small pendulum-angle amplitude is smaller, while under the large initial excitation $\left(10^{\circ}\right)$, the shimmy with large pendulum-angle amplitude is larger. It can be seen from Fig. 8 that, at a speed of $70 \mathrm{~km} / \mathrm{h}$, multi limit -cycles shimmy occurs in the dual-axle steering mechanism under different initial excitations, and the system is in an extremely unstable state.

In order to investigate the effects of road adhesion coefficients on the multiple limit-cycles shimmy characteristics of dual-axle steering mechanism, the road adhesion coefficient $\left(\mu_{1}=\mu_{2}=\mu_{3}=\mu_{4}\right)$ is changed to 0.6, 0.5, 0.4 respectively.Fig. 10-12 show the global bifurcation diagrams of swing angles of the first and second axle versus speed under different road adhesion coefficients.

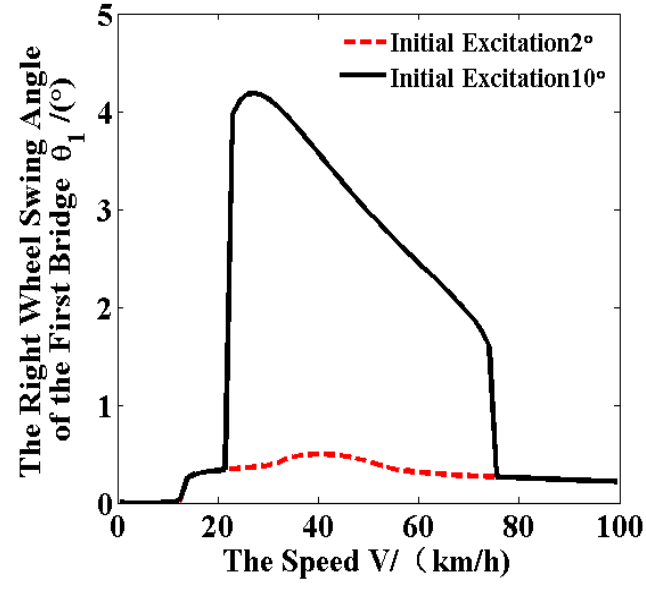

(a) right wheel of the first axle

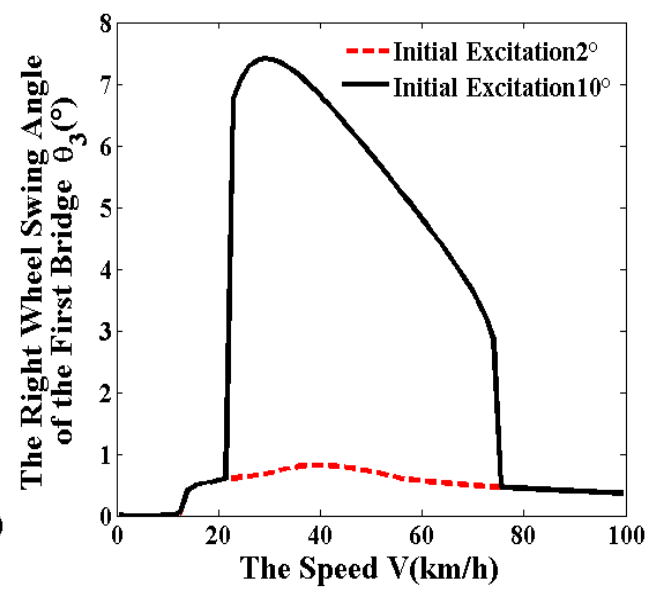

(b) right wheel of the second axle

Fig.10. Bifurcation diagrams of the shimmy system when $\mu_{1}=\mu_{2}=\mu_{3}=\mu_{4}=0.6$ 


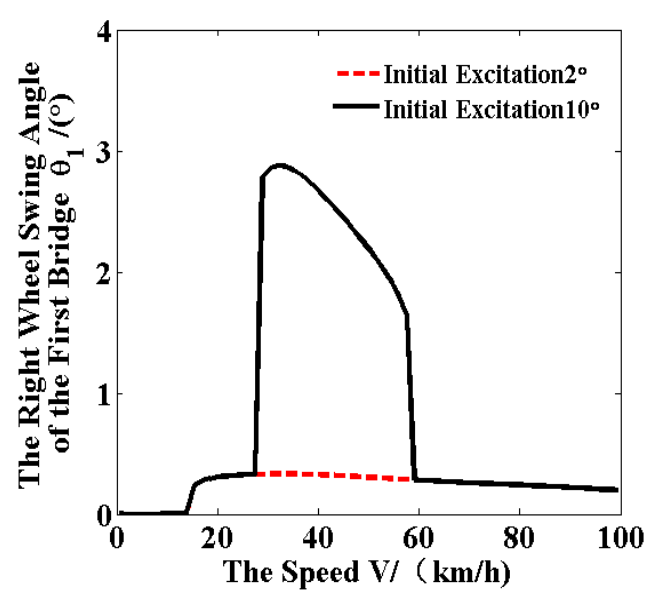

(a) right wheel of the first axle

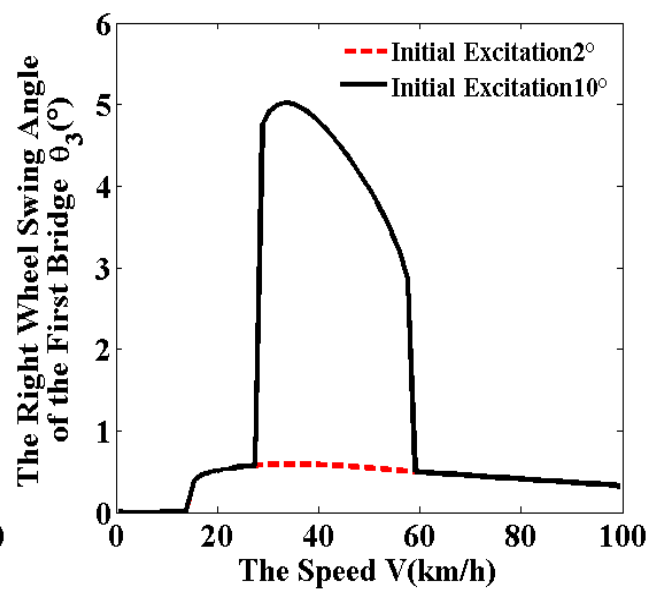

(b) right wheel of the second axle

Fig.11. Bifurcation diagrams of the shimmy system when $\mu_{1}=\mu_{2}=\mu_{3}=\mu_{4}=0.5$

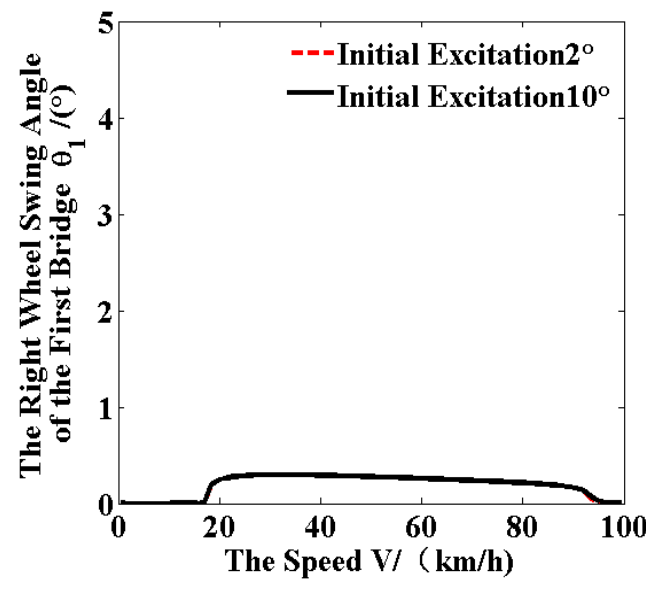

(a) right wheel of the first axle

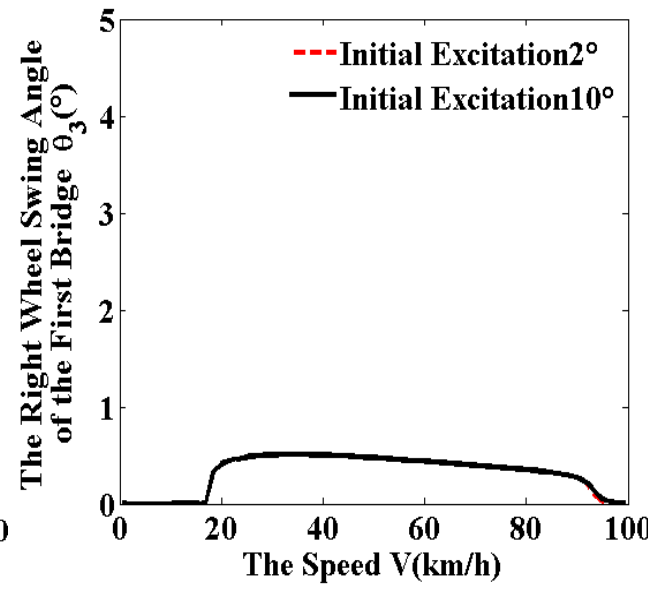

(b) right wheel of the second axle

Fig.12. Bifurcation diagrams of the shimmy system when $\mu_{1}=\mu_{2}=\mu_{3}=\mu_{4}=0.4$

Table.3. Maximum swing angles and the speed ranges of multiple limit-cycles shimmy occurring under

\begin{tabular}{|c|c|c|c|c|}
\hline $\begin{array}{c}\mu_{1}=\mu_{2} \\
=\mu_{3}= \\
\mu_{4}\end{array}$ & $\begin{array}{l}\text { Maximum } \\
\text { swing Angle } \\
\text { of the first } \\
\text { axle }\left(^{\circ}\right)\end{array}$ & $\begin{array}{l}\text { Maximum } \\
\text { swing } \\
\text { Angle of } \\
\text { the second } \\
\text { axle }\left(^{\circ}\right)\end{array}$ & $\begin{array}{l}\text { The speed ranges of } \\
\text { the first axle } \\
\text { multiple limit-cycles } \\
\text { shimmy } \\
\text { occurring }(\mathrm{km} / \mathrm{h})\end{array}$ & $\begin{array}{l}\text { The speed ranges of } \\
\text { the second axle } \\
\text { multiple limit-cycles } \\
\text { shimmy } \\
\text { occurring }(\mathrm{km} / \mathrm{h})\end{array}$ \\
\hline 0.6 & 4.185 & 7.411 & {$\left[\begin{array}{ll}21.5 & 75.5\end{array}\right]$} & {$\left[\begin{array}{ll}21.5 & 75.5\end{array}\right]$} \\
\hline 0.5 & 2.879 & 5.021 & {$\left[\begin{array}{ll}27.5 & 59\end{array}\right]$} & {$\left[\begin{array}{ll}27.5 & 59\end{array}\right]$} \\
\hline 0.4 & 0.295 & 0.505 & disappear & disappear \\
\hline
\end{tabular}

From Fig.10-12 we can get the maximum swing angles and the speed ranges of multiple limit-cycles shimmy occurring under different road adhesion coefficients, as show in Table.3.From the Table.3,It can be concluded that the maximum swing angles of first and second axle decrease with the road adhesion coefficient decreasing. The speed range in which multiple limit-cycles shimmy occurs decreases with the decrease of road adhesion coefficient. And when road adhesion coefficient $\left(\mu_{I}=\mu_{2}=\mu_{3}=\mu_{4}\right)$ reduced to 0.4 , the phenomenon of multiple limit-cycles shimmy disappeared. And the amplitude of the shimmy is very small, that is, it is not easy to 
cause the shimmy phenomenon when the adhesion coefficient is low. In addition, as the road adhesion coefficient decreases, the speed range of multiple limit-cycles occurring tend to shrink to the middle speed range $(40-50 \mathrm{~km} / \mathrm{h})$ which is multi-axle vehicles often travelling. The occurrence of multiple limit-cycles shimmy will seriously affect the handling stability and safety of vehicles. Therefore, when considering the characteristics of multiple limit-cycles shimmy we should not only consider the maximum swing angle, but also consider the speed range of multiple limit-cycles shimmy occurring.

\subsection{Effect of different road adhesion coefficients of the first and second axle on the multiple limit-cycles shimmy characteristics}

In the last section, it is assumed that the road adhesion coefficients of the first and second axle are the same, but it is often not the case in actual driving conditions. So in this section, the effect which taking different road adhesion coefficients of the first and second axle have on the multiple limit-cycles shimmy characteristics is discussed.

Taking the road adhesion coefficients of first axle $\mu_{1,} \mu_{2}\left(\mu_{1}=\mu_{2}\right)$ as 0.6 and keeping unchanged, the road adhesion coefficients of second axle $\mu_{3,} \mu_{4}\left(\mu_{3}=\mu_{4}\right)$ is changed to 0.8 and 0.4 respectively.Fig. 13-14 show the global bifurcation diagrams of swing angles of the first and second axle versus speed.

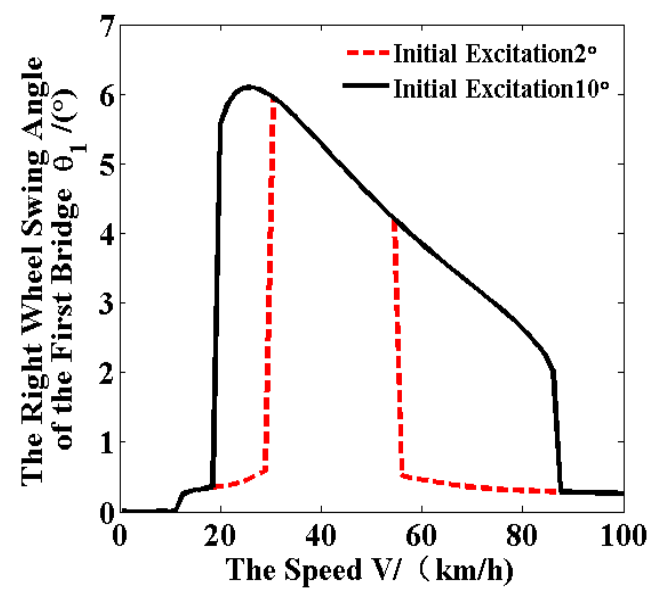

(a) right wheel of the first axle

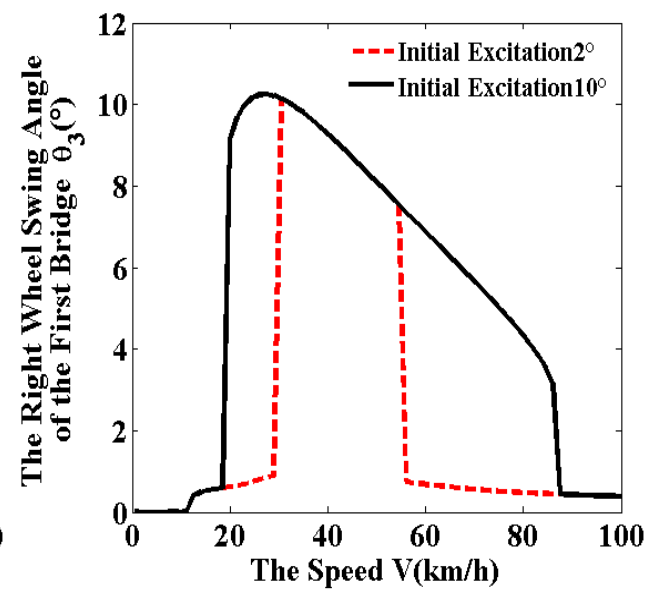

(b) right wheel of the second axle

Fig.13. Bifurcation diagrams of the shimmy system when $\mu_{I}=\mu_{2}=0.6, \mu_{3}=\mu_{4}=0.8$

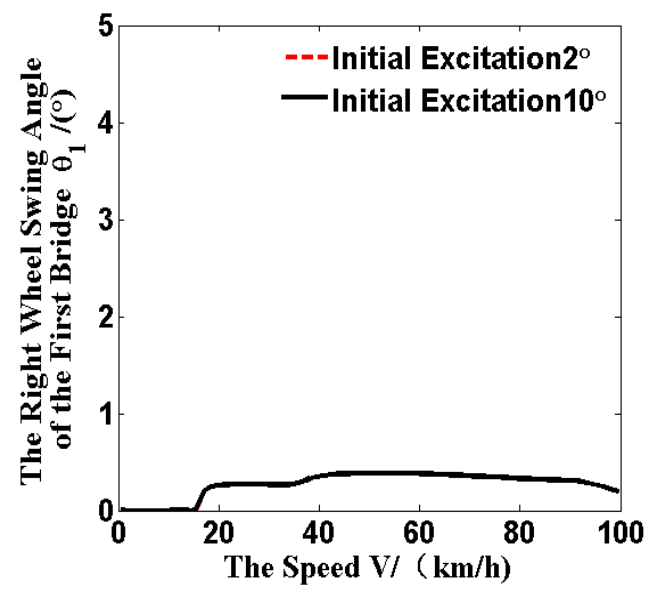

(a) right wheel of the first axle

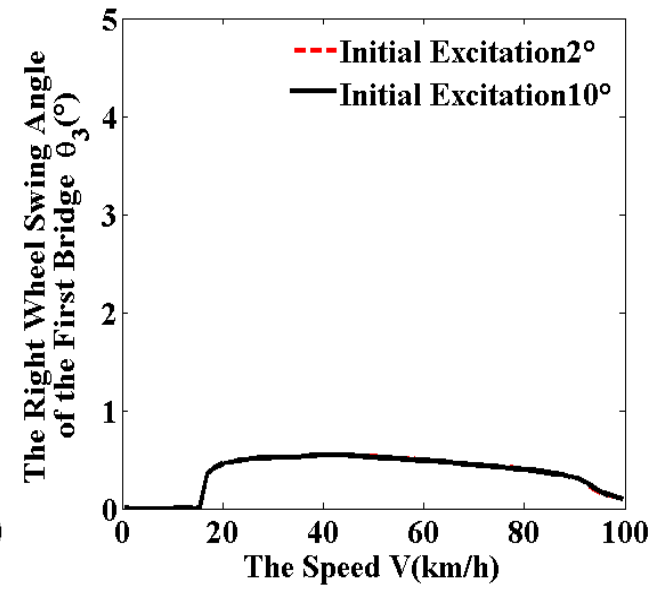

(b) right wheel of the second axle 
Fig.14. Bifurcation diagrams of the shimmy system when $\mu_{I}=\mu_{2}=0.6, \mu_{3}=\mu_{4}=0.4$

Taking the road adhesion coefficients of second axle $\mu_{3}, \mu_{4}\left(\mu_{3}=\mu_{4}\right)$ as 0.6 and keeping unchanged, the road adhesion coefficients of first axle $\mu_{1}, \mu_{2}\left(\mu_{1}=\mu_{2}\right)$ is changed to 0.8 and 0.4 respectively.Fig. $15-16$ show the global bifurcation diagrams of swing angles of the first and second axle versus speed.

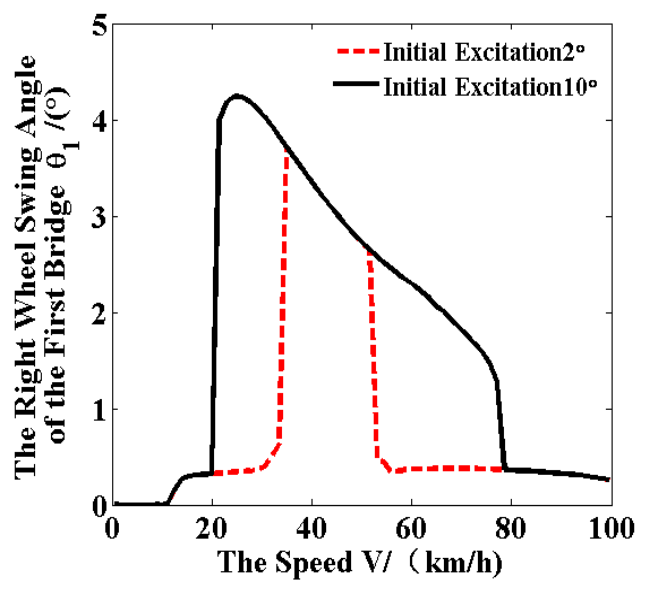

(a) right wheel of the first axle

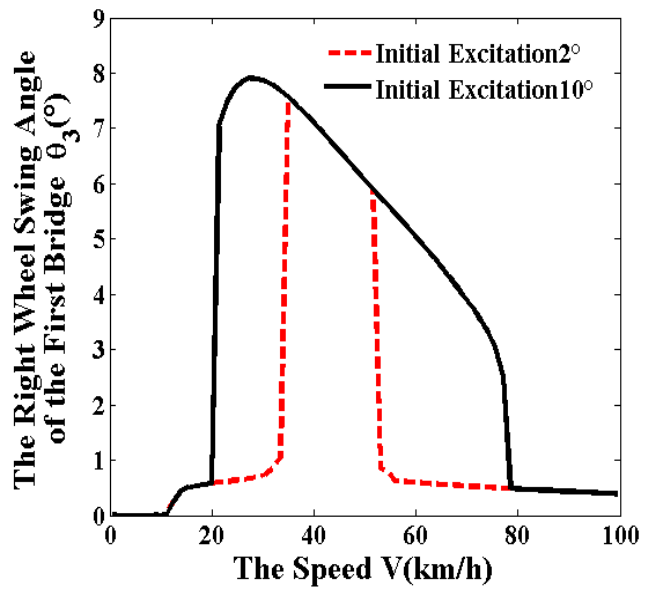

(b) right wheel of the second axle

Fig.15. Bifurcation diagrams of the shimmy system when $\mu_{3}=\mu_{4}=0.6, \mu_{1}=\mu_{2}=0.8$

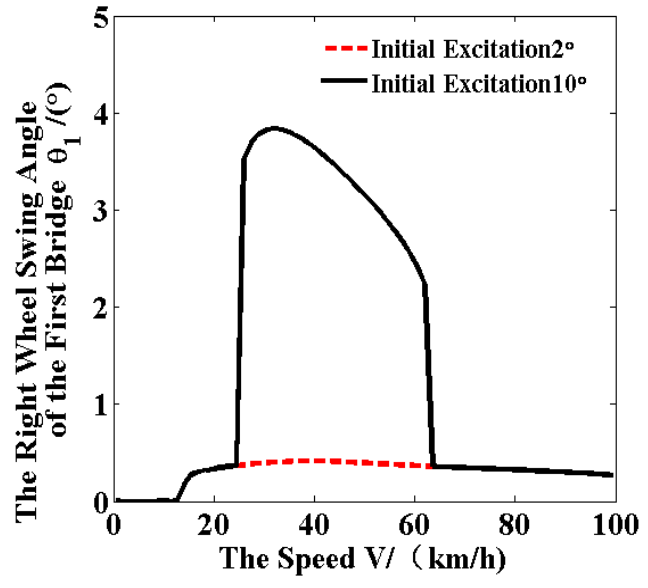

(a) right wheel of the first axle

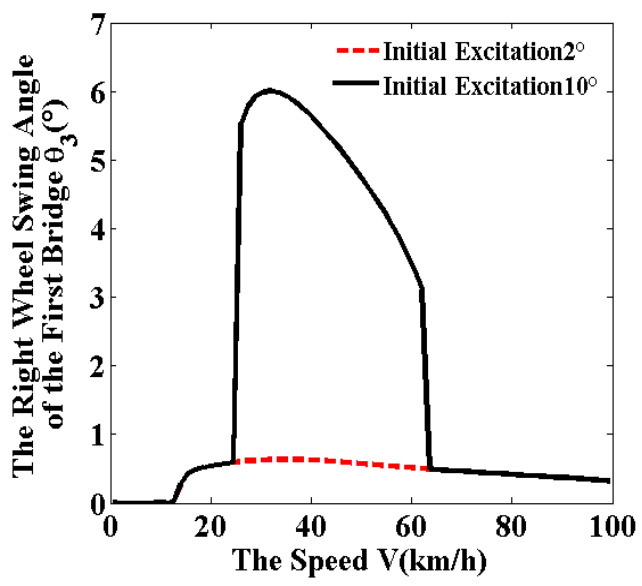

(b) right wheel of the second axle

Fig.16. Bifurcation diagrams of the shimmy system when $\mu_{3}=\mu_{4}=0.6, \mu_{1}=\mu_{2}=0.4$

Table.4. Maximum swing angles and the speed ranges of multiple limit-cycles shimmy occurring under

\begin{tabular}{ccccc}
\multicolumn{5}{c}{ different $\mu_{3}, \mu_{4}\left(\mu_{3}=\mu_{4}\right)$ when $\mu_{I}=\mu_{2}=0.6$} \\
\hline$\mu_{3}=\mu_{4}$ & $\begin{array}{c}\text { Maximum } \\
\text { swing Angle } \\
\text { of the first } \\
\text { axle }\left(^{\circ}\right)\end{array}$ & $\begin{array}{c}\text { Maximum } \\
\text { swing Angle } \\
\text { of the second } \\
\text { axle }\left(^{\circ}\right)\end{array}$ & $\begin{array}{c}\text { The speed ranges of } \\
\text { the first axle multiple } \\
\text { limit-cycles shimmy } \\
\text { occurring }(\mathrm{km} / \mathrm{h})\end{array}$ & $\begin{array}{c}\text { The speed ranges of the } \\
\text { second axle multiple } \\
\text { limit-cycles shimmy } \\
\text { occurring }(\mathrm{km} / \mathrm{h})\end{array}$ \\
\hline 0.8 & 6.098 & 10.25 & {$[18.530 .5],[54.587 .5]$} & {$[18.530 .5],[54.587 .5]$} \\
0.4 & 0.385 & 0.548 & disappear & disappear \\
\hline
\end{tabular}

Table.5. Maximum swing angles and the speed ranges of multiple limit-cycles shimmy occurring under different $\mu_{1}, \mu_{2}\left(\mu_{1}=\mu_{2}\right)$ when $\mu_{3}=\mu_{4}=0.6$ 


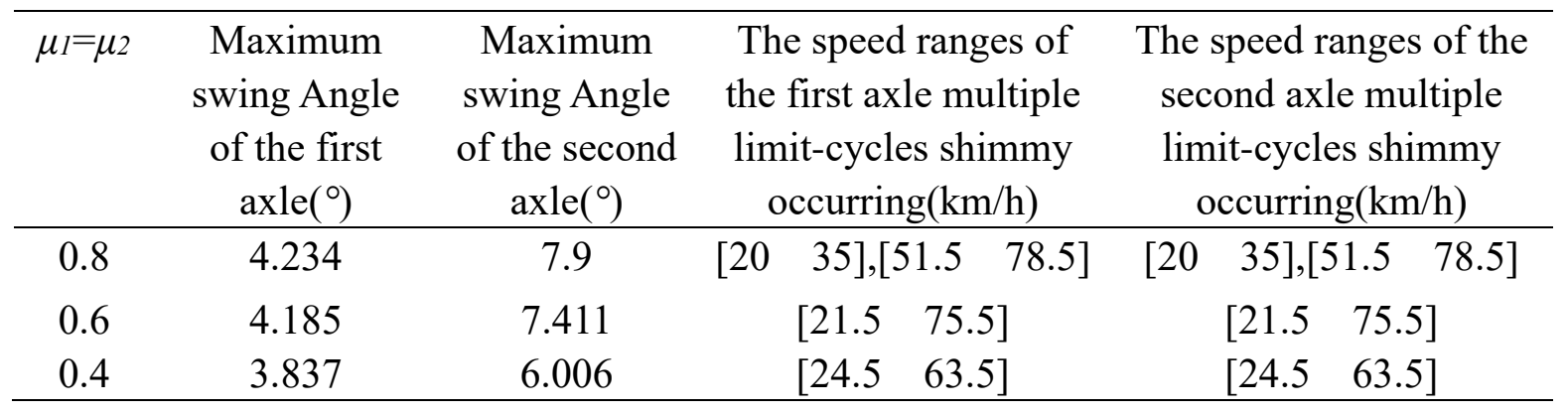

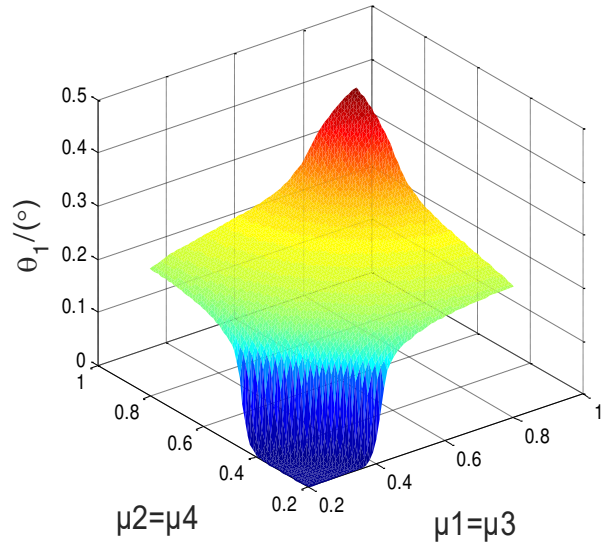

(a)right wheel of the first axle

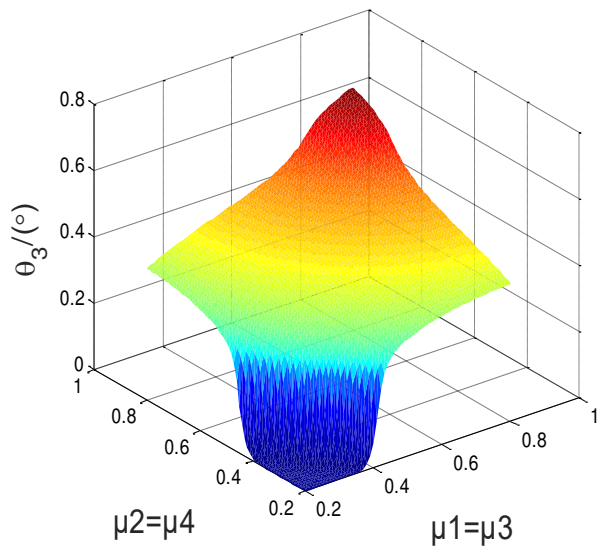

(b) right wheel of the second axle

Fig.17. three-dimensional diagram of the shimmy system

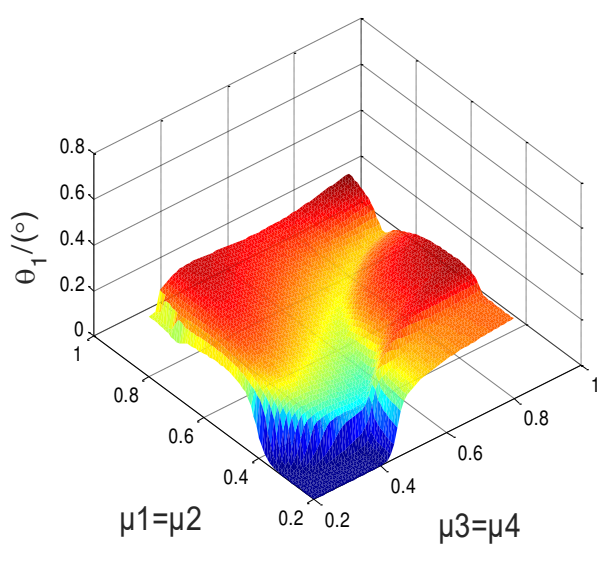

(a) right wheel of the first axle

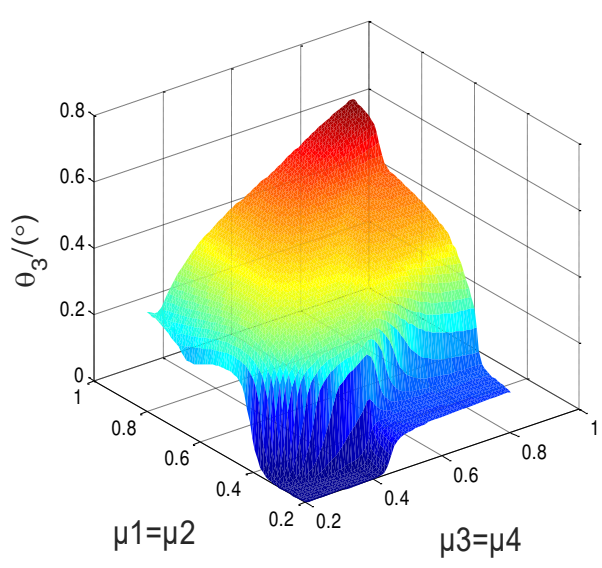

(b) right wheel of the second axle

Fig.18. three-dimensional diagram of the shimmy system

Table. 4 and Table. 5 can be obtained from Fig. 10 and Figs.13-16.Table.4 shows the maximum swing angles and the speed ranges of multiple limit-cycles shimmy occurring under different $\mu_{3,} \mu_{4}\left(\mu_{3}=\mu_{4}\right)$ when $\mu_{1}=\mu_{2}=0.6$. Table. 5 shows the maximum swing angles and the speed ranges of multiple limit-cycles shimmy occurring under different $\mu_{1}, \mu_{2}\left(\mu_{1}=\mu_{2}\right)$ when $\mu_{3}=\mu_{4}=0.6$.

From Table. 4 and Table.5 we can get that keeping the road adhesion coefficients of first or second axle unchanged and changing the road adhesion coefficients of second or first axle respectively, the maximum swing angles and the speed ranges of multiple limit-cycles shimmy occurring decrease with the road adhesion coefficients which is consistent with the conclusions in the previous section.

The comparison of the data in Table 4 and Table 5 shows that the maximum 
shrink angle of the first axle is changed to $\left[6.098^{\circ} 0.385^{\circ}\right]$ and the maximum shrink angle of the second axle is changed to $\left[\begin{array}{ll}10.25^{\circ} & 0.548^{\circ}\end{array}\right]$ when the road adhesion coefficient of the first axle unchanged $\left(\mu_{1}=\mu_{2}=0.6\right)$, while the road adhesion coefficient of the second axle is changed. The phenomenon of multiple limit-cycles

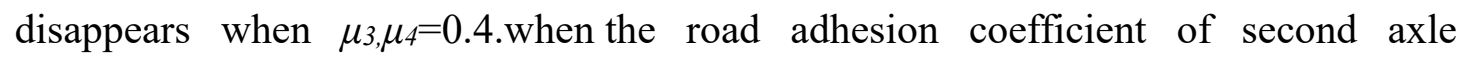
unchanged ( $\left.\mu_{3}=\mu_{4}=0.6\right)$, and the road friction coefficient of the first axle is changed, The maximum shrink angle of the first axle is $\left[4.234^{\circ} 3.837^{\circ}\right]$, and the maximum shrink angle of the second bridge is $\left[\begin{array}{lll}7.9^{\circ} & 6.006^{\circ}\end{array}\right]$.Obviously, when the road adhesion coefficient of the first axle is kept unchanged and the road adhesion coefficient of the second axle is changed, the change interval of the maximum shrink angle of the first axle and the change interval of the velocity of multiple limit cycles are much larger than that of the second axle when the road adhesion coefficient of the first axle is kept unchanged. Fig.13-16 studies the influence of road surface adhesion coefficient of the first axle and the second axle on self-excited shimmy characteristics of multi limit-cycles from the global perspective of velocity. Only a few discrete points are taken, and the influence of continuous change of matching value on self-excited shimmy characteristics of multi-limit cycle cannot be observed.

In order to observe the influence of continuous change of matching value on the shrink characteristics from a global perspective, take $\mathrm{V}=70 \mathrm{~km} / \mathrm{h}$ with an initial excitation of $2^{\circ}$, and draw the three-dimensional diagram of the shrink angle of the right wheel of the first and second bridge on the change of the road adhesion coefficient of the left and right sides of the system and the change of the road adhesion coefficient of the first and second axles, respectively. It can be seen from Figure 17(a) and 17(b) that when $\mu_{1, \mu 3}$ and $\mu_{2}, \mu_{4}$ are large, the shrink angle amplitude of the left/right wheel of the first axle is relatively large. With the decrease of the adhesion coefficient on one side or both sides of the road, the shrink angle amplitude of the left/right wheel of the first axle presents a trend of decrease. From figure 18 (a),18 (b) it can also be observed that the shrink angle of the left/right wheel of the first axle changes on the same pattern, but the road adhesion coefficient of the first axle and the road adhesion of the second axle have different impact on shrink angle, when $\mu_{3}, \mu_{4}$ remain the same and take smaller values, with the decrease of $\mu_{1}, \mu_{2}$ just a little change of the angular amplitude of the right wheel occurs, only appears larger fluctuation when $\mu_{1}, \mu_{2}$ are close to 0.3 . However, when the road adhesion coefficient of the first axle remains unchanged and is at a small value, when $\mu_{3}, \mu_{4}$ decrease to close to 0.4 , the limit-cycles amplitude of the first and second axles has been reduced to a very small value, and the phenomenon of multiple limit-cycles disappears into a single ring shimmy. Based on the above discussion, it can be concluded that the influence of the road adhesion coefficient of the second axle is much greater than that of the first axle. In other words, the characteristics of multi limit-cycles shimmy of dual-axle steering mechanism are more sensitive to the change of adhesion coefficient of the second axle than that of the first axle.

\section{Multi-limit cycle shimmy control of multi-axle steering mechanism}

\subsection{Influence of caster angle of kingpin on shimmy characteristics of multiple}




\section{limit cycles under different road adhesion coefficients}

In order to explore the influence of the caster angle of the kingpin on the shimmy characteristics of multiple limit cycles, different road adhesion coefficients $\left(\mu_{1}=\mu_{2}=\mu_{3}=\mu_{4}\right)$ are taken as $0.6,0.7,0.8$, and the vehicle speed $\mathrm{v}=40 \mathrm{~km} / \mathrm{h}$ to obtain different road adhesion. The partial bifurcation diagram of the swing angle of the right wheel of the first and second axles with the caster angle $\gamma$ under the coefficient. As shown in Figure 19-21.

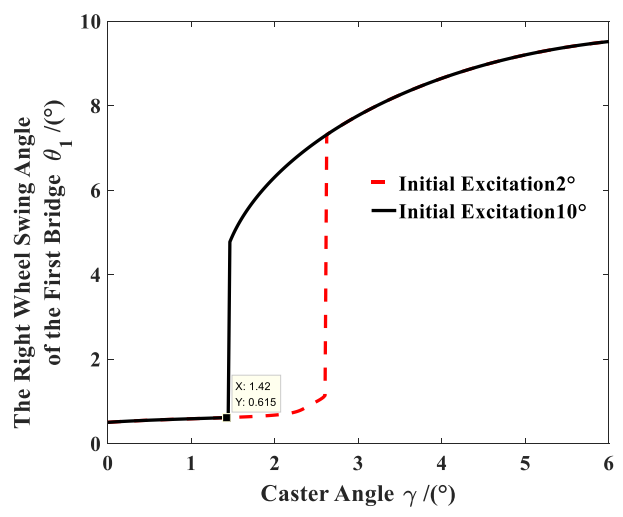

(a) right wheel of the first axle

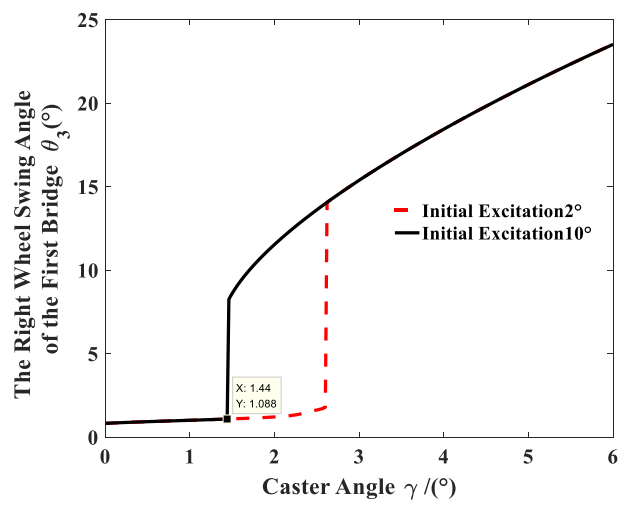

(b) right wheel of the second axle

Fig.19. Bifurcation diagram of steering wheel swing angle when $\mu_{1}=\mu_{2}=\mu_{3}=\mu_{4}=0.6$

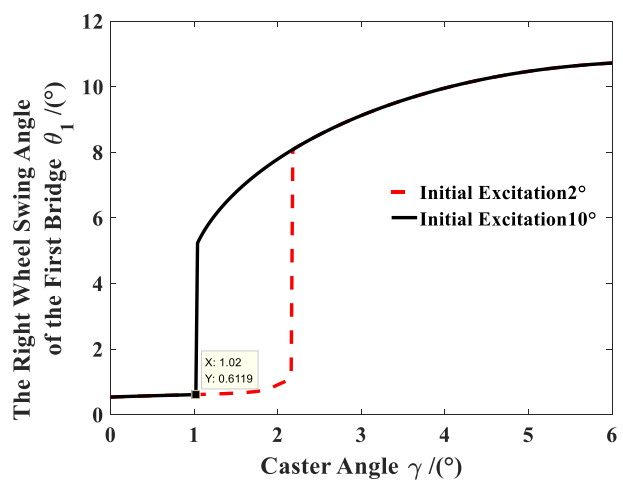

(a) right wheel of the first axle

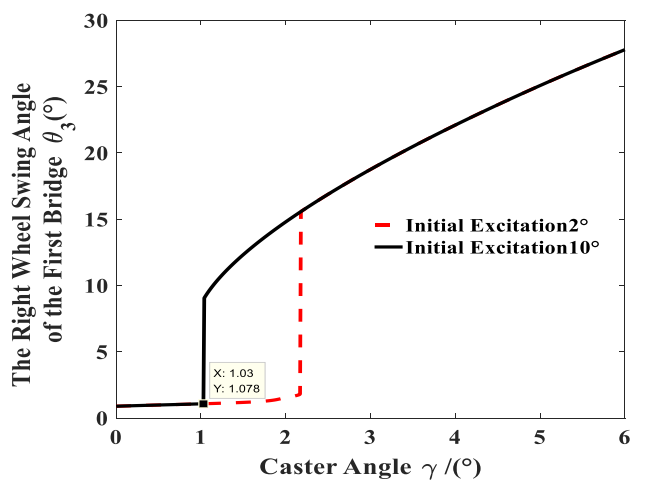

(b) right wheel of the second axle

Fig.20. Bifurcation diagram of steering wheel swing angle when $\mu_{1}=\mu_{2}=\mu_{3}=\mu_{4}=0.7$

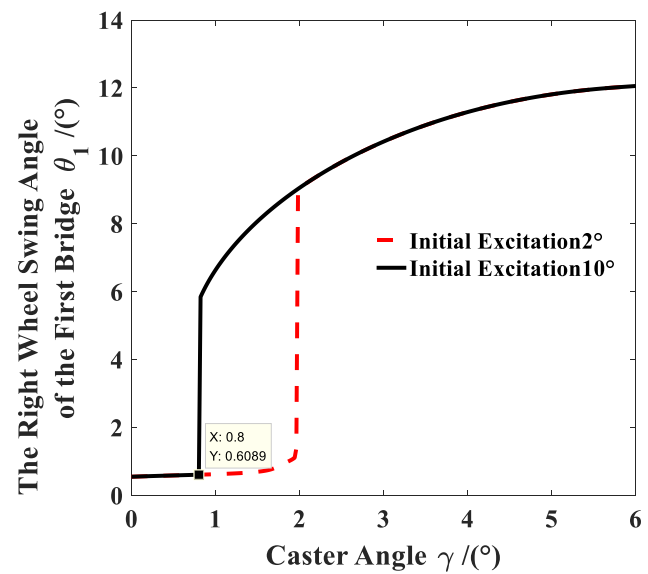

(a) right wheel of the first axle

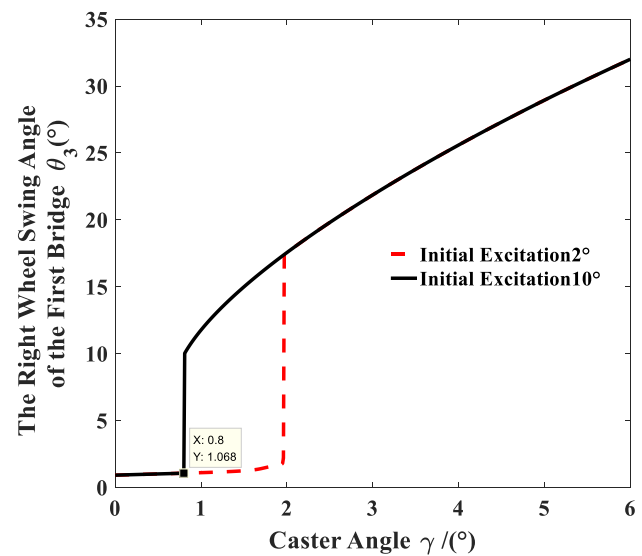

(b) right wheel of the second axle

Fig.21. Bifurcation diagram of steering wheel swing angle when $\mu_{1}=\mu_{2}=\mu_{3}=\mu_{4}=0.8$ 
Table.6. The maximum swing angle of the right wheel and the speed range of multiple limit cycles under different road adhesion coefficients

\begin{tabular}{ccccc}
\hline $\begin{array}{c}\mu_{1}=\mu_{2}= \\
\mu_{3}=\mu_{4}\end{array}$ & $\begin{array}{c}\text { Maximum } \\
\text { swing } \\
\text { angle of } \\
\text { the first } \\
\text { bridge }\left(^{\circ}\right)\end{array}$ & $\begin{array}{c}\text { Maximum } \\
\text { swing } \\
\text { angle of } \\
\text { the second } \\
\text { bridge }\left(^{\circ}\right)\end{array}$ & $\begin{array}{c}\text { Caster angle } \\
\text { range of the first } \\
\text { axle with multiple } \\
\text { limit cycles }\left(^{\circ}\right)\end{array}$ & $\begin{array}{c}\text { Caster angle } \\
\text { range of the } \\
\text { second axle with } \\
\text { multiple limit } \\
\text { cycles }\left({ }^{\circ}\right)\end{array}$ \\
\hline 0.6 & 9.516 & 23.52 & {$\left[\begin{array}{lll}1.44 & 2.62\end{array}\right]$} & {$\left[\begin{array}{lll}1.44 & 2.64\end{array}\right]$} \\
0.7 & 10.72 & 27.76 & {$\left[\begin{array}{lll}1.02 & 2.18\end{array}\right]$} & {$\left[\begin{array}{lll}1.03 & 2.18\end{array}\right]$} \\
0.8 & 12.05 & 32 & {$\left[\begin{array}{lll}0.8 & 1.96\end{array}\right]$} & {$\left[\begin{array}{lll}0.8 & 1.97\end{array}\right]$} \\
\hline
\end{tabular}

From Figure 19-21, the maximum swing angle of the right wheel of the second bridge and the speed range of multiple limit cycles under different road adhesion coefficients are obtained, as shown in Table 6. It can be seen from the table that as the caster angle of the kingpin decreases, the amplitude of the swing angle of the first and second bridges decreases, and the range of the caster angle of the multiple limit cycle occurs to the left as the road adhesion coefficient increases. Side movement; combined with the previous conclusion, that is, the larger the adhesion coefficient, the more likely it is to cause shimmy. It can be seen that the reduction of the caster angle of the kingpin has obvious suppression of the shimmy phenomenon. In addition, there is another phenomenon worth noting. For different road adhesion coefficients, the multiple limit cycle intervals of the first and second bridges are basically the same, that is, as the caster angle decreases, the multiple limit cycle shimmy of the first and second bridges The phenomenon can be eliminated at the same time.

In order to observe the influence of the continuous change of the matching value on the shimmy characteristics from a global perspective, take $\mathrm{v}=40 \mathrm{~km} / \mathrm{h}$, and make the first and second bridges the initial excitation $10^{\circ}$ and $2^{\circ}$ corresponding to the amplitude difference of the right wheel swing angle of the first and second bridges. A three-dimensional graph of the coefficient and the caster angle of the kingpin. As can be seen from the figure 22, the three-dimensional images of the right wheel of bridge one and two are divided into three regions, corresponding to low-amplitude single limit cycle shimmy, multi-limit cycle shimmy and high-amplitude single limit cycle shimmy respectively. In practical application, we not only hope that the steering bridge has small amplitude shimmy, but also hope to adjust the multi-limit ring shimmy to single limit ring shimmy. Therefore, we should match the optimal kingpin backangle in the low amplitude single limit ring shimmy area according to the actual road adhesion coefficient

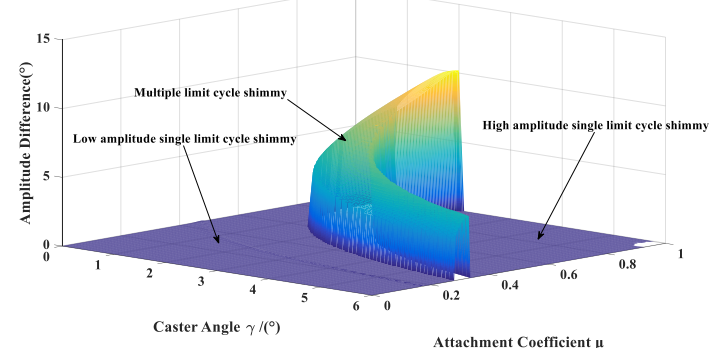

(a) right wheel of the first axle

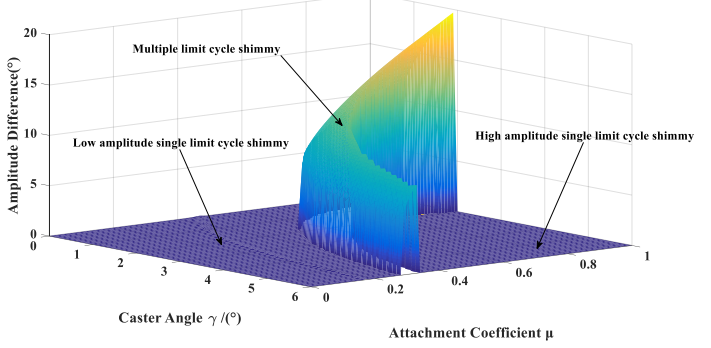

(b) right wheel of the second axle 
Fig.22. A three-dimensional graph of the amplitude difference with the road adhesion coefficient and the caster angle of the kingpin

In the selection of parameters, different values of caster angles of kingpins can be matched according to the change of road adhesion coefficient to obtain smaller steering wheel swing amplitude and smaller multiple limit cycle swing vibration interval. In order to observe the effect of optimization, taking the road adhesion coefficient as 0.6 as an example, the kingpin back Angle is $1.4^{\circ}$ according to the three-dimensional diagram, and the global bifurcation diagram of the right wheel swing Angle of the first and second Bridges is made according to the system parameters at this time.

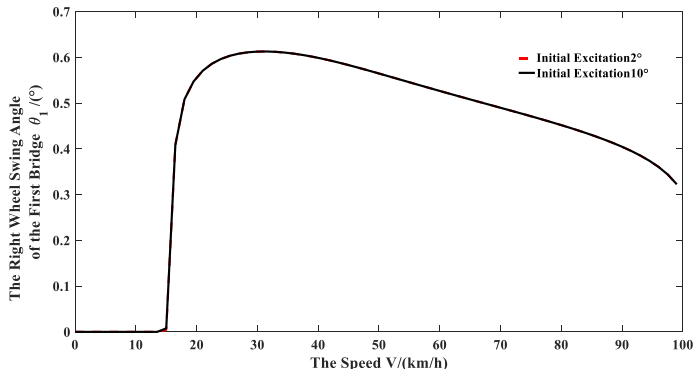

(a) right wheel of the first axle

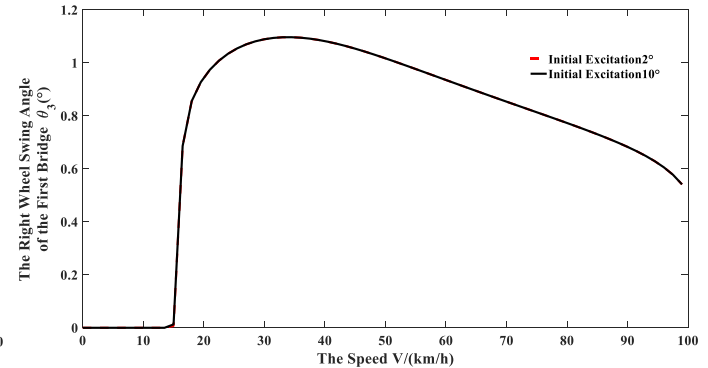

(b) right wheel of the second axle

Fig.23. Bifurcation diagram of the right wheel swing angle of the first and second bridges after optimization

According to Fig.23, compared with Fig. 10, it is found that after adjusting the caster angle of the kingpin, the multi-limit range of the steering wheel shimmy of the first and second axles has disappeared. In addition, it is found that the amplitude of the steering wheel shimmy has been greatly reduced after the adjustment, achieving the purpose of parameter optimization.

\subsection{The influence of the rigidity matching of the transition link on the shimmy characteristics of multiple limit cycles}

Through further research on the system parameters, it is found that the stiffness of the transition link has a great influence on the shimmy characteristics of the steering wheel with multiple limit cycles. Reasonable matching of the appropriate stiffness of the transition link has a very positive effect on eliminating the multi-limit cycle shimmy phenomenon of the steering wheel. Therefore, $\mathrm{v}=40 \mathrm{~km} / \mathrm{h}$, adhesion coefficient is 0.8 , and three-dimensional drawings of the first transition tie rod stiffness $\mathrm{k}_{\mathrm{t} 1}$ and the second transition tie rod stiffness $\mathrm{k}_{\mathrm{t} 2}$ corresponding to the amplitude difference of steering wheel swing Angle corresponding to the initial excitation of $10^{\circ}$ and $2^{\circ}$ of the first and second Bridges are made respectively.

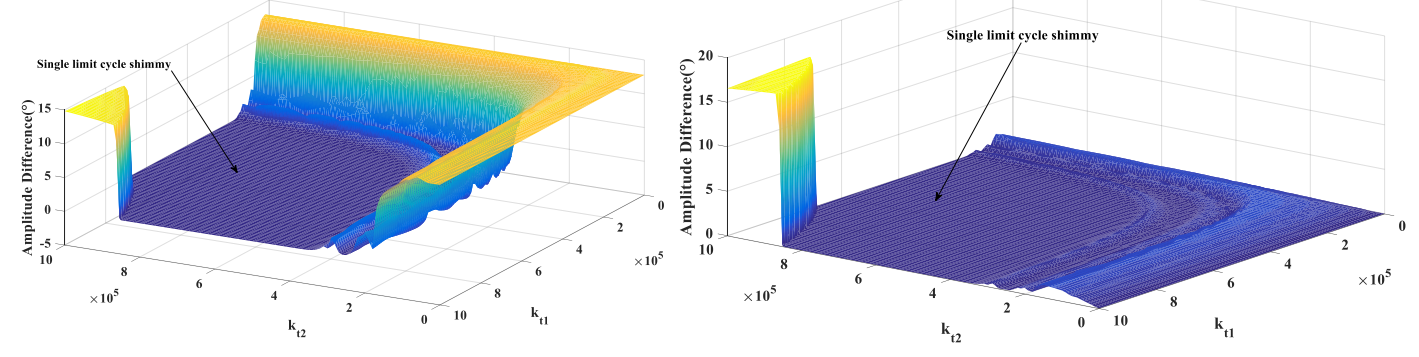



(a) right wheel of the first axle
(b) right wheel of the second axle

Fig.24. A three-dimensional diagram of the amplitude difference under different excitations with the stiffness of the transition link

It can be seen from Figure 24 that by matching the stiffness of different transition rods, the multi-limit cycle shimmy of the steering wheel can be changed to a single limit cycle. When the road adhesion coefficient $\mu=0.8$, the single limit cycle shimmy stiffness intervals of the steering wheel $\mathrm{k}_{\mathrm{t} 1}$ and $\mathrm{k}_{\mathrm{t} 2}$ are both $\left[\begin{array}{ll}4 \times 10^{5} & 8 \times 10^{5}\end{array}\right]$.

\section{5. conclusion}

In this paper, a mathematical model of dual-axle steering mechanism considering the vehicle-road coupling has been established. And the structural parameters and partial stiffness in the mathematical model are determined by one multi-axle vehicle with dual-axle steering mechanism we studied. Under the condition of keeping other parameters unchanged except the road adhesion coefficients, the numerical analysis method is used to discuss the effects of the vehicle-road coupling on multiple limit-cycles shimmy characteristics of dual-axle steering mechanism.

1. Under different initial excitations, the dual-axle steering mechanism produced multiple limit-cycles shrink phenomenon, and the intensity of shrink of the first bridge and the second bridge is inconsistent. In fact, the second axle tire wear of multi-axle vehicles is always greater than the first axle tire wear, which reflects the reliability of the 9-DOF model established in this paper. With the decrease of the adhesion coefficient of the split road, the amplitude of the maximum shimmy Angle of the first axle and the second axle decreases, and the shimmy of the multi-limit cycle is restrained to a certain extent. Similarly, the velocity interval of multi limit-cycles shimmy also decreases, and the velocity interval of multi-limit cycle shrinks towards the intermediate velocity interval of multi-axle vehicles as the adhesion coefficient of the split road decreases.

2. Compared with the change of the adhesion coefficient of the first and second axle, the angle amplitude of wheel shimmy induced by the change of the adhesion coefficient of the second bridge fluctuates greatly, that is, when the road condition changes greatly, the driving stability and safety will be insufficient. Therefore, in the design of multi-axle vehicles, the characteristics of the shimmy varying with the adhesion coefficient of the first axle and the second axle can be taken as a reference to enhance the steering stability of multi-axle vehicles.

3. The system parameters that have a greater impact on the shimmy phenomenon, namely, the caster angle of the kingpin and the stiffness of the transition link are found, and the optimal interval of the corresponding parameters is analyzed. After selecting the appropriate parameters, it is found that not only the steering wheel shimmy The amplitude has been significantly reduced, and the phenomenon of multi-limit cycle shimmy has disappeared.

\section{Data availability}

All data generated or analyzed during this study are included in this published article.

\section{Acknowledgment}


This project was supported by National Natural Science Foundation of China(Grant NO.51375130,51050002,51875154)

Some parameters of the double-axle steering system in this paper are selected from the article of Professor Wei Daogao[17], thanks to the help of Professor Wei Daogao.

\section{References}

[1] K. Watanabe, J. Yamakawa, M. Tanaka, T. Sasaki, Turning characteristics of multi-axle vehicles, J Terramechanics, 44 (2007) 81-87.

[2] C. Mu, J. Yu, Y. Yang, K. Wu, Design for dual-front axle steering angle of the heavy truck, in: 2010 International Conference on Educational and Network Technology, 2010, pp. 185-187.

[3] J. Stuart, S. Cassara, B. Chan, N. Augustyniak, Recent Experimental and Simulation Efforts to Mitigate Wobble and Shimmy in Commercial Line Haul Vehicles, in, SAE International, 2014.

[4] H.B. Pacejka, Analysis of the Shimmy Phenomenon, Proceedings of the Institution of Mechanical Engineers: Automobile Division, 180 (1965) 251-268.

[5] S. Li, Y. Lin, Study on the bifurcation character of steering wheel self-excited shimmy of motor vehicle, Vehicle System Dynamics, 44 (2006) 115-128.

[6] J. Lu, Y. Xu, C. Hu, A.F. Vakakis, L.A. Bergman, 5-DOF Dynamic Model of Vehicle Shimmy System with Clearance at Universal Joint in Steering Handling Mechanism, Shock and Vibration, 20 (2013) 469497.

[7] D. Wei, Y. Wang, T. Jiang, S. Zheng, W. Zhao, Z. Pan, Chaos vibration of pinion and rack steering trapezoidal mechanism containing two clearances, Mechanical Systems and Signal Processing, 92 (2017) 146-155.

[8] Y. Hou, Y. Hu, D. Hu, C. Li, Synthesis of Multi-Axle Steering System of Heavy Duty Vehicle Based on Probability of Steering Angle, in, SAE International, 2000.

[9] D.H. Wu, J. Hai, Analysis of dynamic lateral response for a multi-axle-steering tractor and trailer, International Journal of Heavy Vehicle Systems, 10 (2003) 281-294.

[10] D.E. Williams, Generalised multi-axle vehicle handling, Vehicle System Dynamics, 50 (2012) 149-166.

[11] M. Demić, Analysis of Influence of Design Parameters on Steered Wheels Shimmy of Heavy Vehicles, Vehicle System Dynamics, 26 (1996) 343-379.

[12] X. Chen, N. Xu, K. Guo, Tire wear estimation based on nonlinear lateral dynamic of multi-axle steering vehicle, International Journal of Automotive Technology, 19 (2018) 63-75.

[13] J. Ding, K. Guo, Development of a generalised equivalent estimation approach for multi-axle vehicle handling dynamics, Vehicle System Dynamics, 54 (2016) 20-57.

[14] T. Mi, G. Stepan, D. Takacs, N. Chen, Shimmy model for electric vehicle with independent suspensions, Proceedings of the Institution of Mechanical Engineers, Part D: Journal of Automobile Engineering, 232 (2017) 330-340.

[15] X. Li, N. Zhang, X. Jin, N. Chen, Modeling and Analysis of Vehicle Shimmy with Consideration of the Coupling Effects of Vehicle Body, Shock and Vibration, 2019 (2019) 3707416.

[16] D. Wei, K. Xu, Y. Jiang, C. Chen, W. Zhao, F. Zhou, Hopf Bifurcation Characteristics of Dual-Front Axle Self-Excited Shimmy System for Heavy Truck considering Dry Friction, Shock and Vibration, 2015 (2015) 839801.

[17] W. Daogao, Z. Wei, Z. Wenhao, Z. Yinjie, W. Wei, J. Ping, W. Bofu, J. Hao, L. Jun, Y. Andong, J. Gensheng, Multiple limit-cycles shimmy characteristics of dual-axle steering mechanism considering the wheel alignment parameters, Proceedings of the Institution of Mechanical Engineers, Part C: Journal of Mechanical Engineering Science, 234 (2020) 3360-3379. 
[18] H. Du, Z. Wang, Y. Wang, H. Huang, Adaptive Robust Control of Multi-Axle Vehicle Electro-Hydraulic Power Steering System With Uncertain Tire Steering Resistance Moment, IEEE Access, 7 (2019) 5519-5530.

[19] H. Du, Q. Zhang, S. Chen, J. Fang, Modeling, simulation, and experimental validation of electro-hydraulic power steering system in multi-axle vehicles, Proceedings of the Institution of Mechanical Engineers, Part D: Journal of Automobile Engineering, 233 (2017) 317-332.

[20] B. Li Chun, D. Pavelescu, The friction-speed relation and its influence on the critical velocity of stick-slip motion, Wear, 82 (1982) 277-289.

[21] H. Pacejka, I.J.M. Besselink, Tire and Vehicle Dynamics, Elsevier Science \& Technology, Oxford, UNITED KINGDOM, 2012.

[22] E. Bakker, L. Nyborg, H.B. Pacejka, Tyre Modelling for Use in Vehicle Dynamics Studies, in, SAE International, 1987.

[23] S.-B. Lu, Y.-N. Li, S.-B. Choi, L. Zheng, M.-S. Seong, Integrated control on MR vehicle suspension system associated with braking and steering control, Vehicle System Dynamics, 49 (2011) 361-380.

[24] Qian, Z. S. Study of steering wheel shimmy for vehicle with independent front suspension (in Chinese). Master Thesis, Tsinghua University, Beijing, China. (1995)

\section{Declaration}

\section{Conflict of interest:}

The authors declared no potential conflicts of interest with respect to the research, author- ship, and/or publication of this article. 